\title{
Early exercise premium method for pricing American options under the J-model
}

Yacin Jerbi(i)

\author{
Correspondence: \\ Yacin.Jerbi@gmail.com \\ Department of Computer Sciences \\ and Applied Mathematics, Ecole \\ Nationale des Ingénieurs de Sfax, \\ BPW 3038 Sfax, Tunisia
}

\begin{abstract}
Background: This study develops a new model called $J$-am for pricing American options and for determining the related early exercise boundary (EEB). This model is based on a closed-form solution J-formula for pricing European options, defined in the study by Jerbi (Quantitative Finance, 15:2041-2052, 2015). The J-am pricing formula is a solution of the Black \& Scholes (BS) PDE with an additional function called $f$ as a second member and with limit conditions adapted to the American option context. The aforesaid function $f$ represents the cash flows resulting from an early exercise of the option.
\end{abstract}

Methods: This study develops the theoretical formulas of the early exercise premium value related to three American option pricing models called J-am, BS-am, and Heston-am models. These three models are based on the J-formula by Jerbi (Quantitative Finance, 15: 2041-2052, 2015), BS model, and Heston (Rev Financ Stud, 6:327-343, 1993) model, respectively. This study performs a general algorithm leading to the EEB and to the American option price for the three models.

Results: After implementing the algorithms, we compare the three aforesaid models in terms of pricing and the EEB curve. In particular, we examine the equivalence between $J$-am and Heston-am as an extension of the equivalence studied by Jerbi (Quantitative Finance, 15:2041-2052, 2015). This equivalence is interesting since it can reduce a bi-dimensional model to an equivalent uni-dimensional model.

Conclusions: We deduce that our model $J$-am exactly fits the Heston-am one for certain parameters values to be optimized and that all the theoretical results conform with the empirical studies. The required CPU time to compute the solution is significantly less in the case of the J-am model compared with to the Heston-am model.

Keywords: American option pricing, Stochastic volatility model, Early exercise boundary, Early exercise premium, J-law, J-process, J-formula, Heston model

\section{Background}

The valuation of American options, while a challenge, is of interest to both academics and traders. American options are more common than their European counterparts; they allow more flexibility since they can be exercised at any time between the current time and maturity. They are presented as a compound option that includes a European option and an early exercise premium (EEP). Hence, their prices are higher than those of European options, and they are more complicated to be modeled. These prices are also significantly affected by the volatility level. The studies by Ju (1998) and Detemple and Rindisbacher (2005) regarding the American option pricing models are based on the Black and Scholes (1973) model and then cannot explain the reality of the financial

(C) The Author(s). 2016 Open Access This article is distributed under the terms of the Creative Commons Attribution 4.0 International License (http://creativecommons.org/licenses/by/4.0/), which permits unrestricted use, distribution, and reproduction in any medium, provided you give appropriate credit to the original author(s) and the source, provide a link to the Creative Commons license, and indicate if changes were made. 
markets, particularly the smile of volatility. Since the dynamics of volatility are fundamental in elaborating trading strategies for hedging and arbitrage, the pricing of the option under stochastic volatility model is important. The introduction of an additional stochastic volatility factor enormously complicates the pricing of the American options. Currently, this can be done only through numerical schemes, which involve solving integral equations as in the studies by Kim (1990), Huang et al. (1996), Sullivan (2000), Detemple and Tian (2002); performing Monte Carlo simulations as in the studies by Broadie and Glasserman (1997), Longstaff and Schwartz (2001), Rogers (2002), Haugh and Kogan (2004); or discretizing the partial differential equation as in the works of Brennan and Schwartz (1977), Clarke and Parrott (1999), and Ikonen and Toivanen (2007). The majority of the studies performed in this area are based on a numerical resolution of the problem using either parametric or nonparametric models. Currently, both academicians and practitioners need a general closed-form solution as a theoretical reference for pricing American option models, considering volatility as stochastic. Such a model is useful for interpreting the results of other models. Moreover, it enables the understanding of the logic behind the option pricing process. The pricing of an American call or put has no explicit closed-form solution. This is because the optimal boundary above which the American call will be exercised is unknown and is a part of the option price solution. Therefore, efforts have been focused on developing a numerical approximation scheme, allowing for a pricing of the American option that is more accurate as well as a faster one than the lattice- or simulation- based methods that are time consuming and computationally more demanding. These schemes are based on integral representations of the American option evaluation formula and exploit the PDE satisfied by the option price. Until now, several studies on European option pricing using stochastic volatility models that lead to a closed-form solution have been conducted. These include studies by Hull and White (1987), Wiggins (1987), Stein and Stein (1991), Heston (1993), and Carr and Wu (2004). The most important study is that by Heston (1993), who considered volatility as stochastic to introduce the skewness and kurtosis effects and ensured that the model fits the reality of the financial market ("smile curve"). He considered two state variables: the underlying asset price and volatility. Jerbi (2015) developed a closed form solution $J$-formula based on the $J$-process considering the skewness and kurtosis effects as well as the smile effect. He showed that for the given parameter values related to the Heston volatility process, there exists a $\left(\lambda^{*}, \theta^{*}\right)$ that ensures the equivalence of the two models by minimizing the gap between the generated prices. He then matched the Heston results with a mono-dimensional model than with a bi-dimensional one. This study extends this equivalence to American options. The price of an American option is the sum of the related European option and the EEP called $\varepsilon$. If the European option value is a solution of a PDE, then the American option value is a solution of the same PDE with a second member equal to a function $f$ instead of zero (PDE- $f$ ). This function depends on the EEB (to be determined). It is the difference between the cash flows generated by the early exercise of the American option. In short, we can say that the European option price Ve is the solution for the homogeneous PDE and that the EEP $\varepsilon$ is a particular solution for the PDE-f. Hence, the American option price $\mathrm{Va}=\mathrm{Ve}+\varepsilon$ constitutes the general solution of the PDE-f. In Heston's (1993) model, the aforesaid PDE is the Garman PDE (1976). In the case of the $J$-model, the PDE is the BS PDE, based on a dynamics of the underlying asset price under the $J$-process (please refer to Jerbi (2015)). The function $\mathrm{f}$ is the same for the 
two models. The EEP value $\varepsilon$ is the sum of the cash flows expectancies between the current time and maturity discounted at the current time. Those cash -flows are generated due to the early exercise of the option, and their calculation is based on the function $f$. In both cases, the solution of the PDE-f is based on the optimal EEB as an optimal limit for exercising the American option. The pricing of the option relies on the determination of such a boundary. The $J$-process is an extension of the Wiener process, by considering the skewness and kurtosis effects. The use of the J-model instead of the Heston's (1993), as an equivalent American option pricing model, makes the solution simpler and easier to interpret. Besides, it significantly improves the time consumed (CPU time) for a given accuracy.

This study first presents the expression of the $J$-formula elaborated by Jerbi (2015). We then elaborate (in detailed calculus) the expression of the EEP $\varepsilon$, related to $J$-am model. Second, we deduce the one related to the BS model by setting parameter $\lambda$ equal to zero. Third, we deduce the expression of the EEP related to the Heston-am model, with a rate of dividend distribution equal to $\mathrm{Q}$. Then, we detail the general methodology to determine, step by step, both the price of the American option and the limit values of the underlying asset belonging to the EEB. The determination of some points of the EEB is time consuming. In fact, these points are sufficient to determine the EEB, with good accuracy, in the form of a polynomial. This considerably reduces the time for calculating the price of an American option. This methodology can be applied to both the $J$-am and Heston-am models. In empirical studies, we compare the results of the two models in terms of accuracy and CPU time. This comparison helps examine the equivalence of the two models such as we can reduce the bi-dimensional model of Heston-am to a unidimensional model fitting the reality of the financial market, by including the skewness and kurtosis effects. This equivalence reduces the CPU time under an equivalent accuracy. After studying this equivalence, we focus on the $J$-am model to examine the effects of its inputs and parameters on the related option price.

\section{Methods}

\section{Snell envelop and early exercise of option}

For an American option, the EEB is defined by the curve $S^{*}(\tau)$, representing the early exercise option limit value of the underlying asset at each time to maturity $\tau$ (please refer to Kwok (1998)). From this limit value, the early exercise of the option becomes interesting.

Let us denote the value of the American call by $\mathrm{C}$, its strike price by $\mathrm{K}$, and its early exercise limit value belonging to the EEB by $S_{c}^{*}(\tau)$; this call will be exercised if we have $S$ $(\tau)=S_{c}^{*}(\tau)$. Then, $C\left(S_{c}^{*}(\tau), \tau\right)=S_{c}^{*}(\tau)-K$ and $\left[\frac{\partial C}{\partial S}\right]_{S=S_{c}^{*}(\tau)}=1$. If we have $S(\tau)<S_{c}^{*}(\tau)$, the call will not be exercised.

However, if $S(\tau) \geq S_{c}^{*}(\tau)$, the call will be exercised. We buy the underlying asset whose value is $S$ (which generates a dividend with a rate $Q$ ) and disburse the amount $K$ (which generates interest with a rate $r$ ). Hence, the early exercise of the call induces a cash flow $Q S-r K$.

Let us denote the value of the American put by $\mathrm{P}$ and its Snell envelop by $S_{p}^{*}(\tau)$, this put will be exercised if we have $S(\tau)=S_{p}^{*}(\tau)$. In this case, $P\left(S_{p}^{*}(\tau), \tau\right)=K-S_{p}^{*}(\tau)$ and $\left[\frac{\partial P}{\partial S}\right]_{S=S_{p}^{*}(\tau)}=-1$.

If $S(\tau) \leq S_{p}^{*}(\tau)$, the put will be exercised. Therefore, we sell the underlying asset and cash an amount K. Hence, the early exercise of the put induces a cash flow equal to $r K-Q S$; if $S(\tau)>S_{p}^{*}(\tau)$, the put will not be exercised. This is summarized in Table 1 . 
Table 1 The function $\mathrm{f}$ of the generated cash-flow in case of the option early exercise or not

\begin{tabular}{lll}
\hline & $C A L L$ & PUT \\
\hline$S \leq S^{*}$ & $f(S, \tau)=0$ & $f(S, \tau)=r K-Q S$ \\
$S>S^{*}$ & $f(S, \tau)=Q S-r K$ & $f(S, \tau)=0$ \\
\hline
\end{tabular}

The EEB, as plotted in Fig. 1, is the set $S_{c}^{*}(\tau)$ for the call and $S_{p}^{*}(\tau)$ for the put, when the time to maturity $\tau$ varies. Regarding the symmetry, in considering the exchange between the underlying asset and liquidity with their respective return rates $Q$ and $r$, the prices of an American call $C$ and an American put $P$, with an underlying price $S$, with a strike price $K$, with time to maturity $\tau$, and with volatility $\sigma$, are such that $C(S, \tau, K, r, Q, \sigma)=P(K, \tau, S, Q, r, \sigma)$.

The related EEB is such that $S_{C}^{*}(\tau, r, Q) * S_{P}^{*}(\tau, Q, r)=K^{2}(\operatorname{see} \operatorname{Kwok}(1998))$.

\section{American option pricing model based on the $J$-formula as a uni-dimensional approach}

Our proposed American option pricing model, named $J$-am, is based on the $J$-formula developed in Jerbi (2015). For the European pricing model, we consider the same hypothesis used in BS with the only difference that the underlying asset price is supposed to follow the $J$-process defined as follows: $\frac{d S_{t}}{S_{t}}=\mu d t+\sigma d z_{t}$, where $\mu$ and $\sigma$ are constants and $d z_{t}=U_{t} \sqrt{d t}$, where $U_{t}=\frac{\left[W_{t}-E_{W}\right]}{\overline{\sigma_{W}}}$ with $W_{t}$ follows the $J$-Law: $\left(W_{t} \rightarrow J(\lambda, \theta)\right)$ defined in the study by Jerbi (2011) by its probability density function $g(w, \lambda, \theta)=\frac{e^{-\frac{1}{2} w^{2}} N(\lambda w+\theta)}{\operatorname{Jer}(\lambda, \theta) \sqrt{2 \pi}}$ with $\operatorname{Jer}(\lambda, \theta)=\int_{-\infty}^{+\infty} \frac{e^{-\frac{1}{2} w^{2}} N(\lambda w+\theta)}{\sqrt{2 \pi}} d w$. The expected value of $W_{t}$ and its standard deviation are $E_{W}=E\left(W_{t}\right)$ and $\sigma_{W}=\sqrt{V\left(W_{t}\right)}$, respectively. As indicated by Jerbi (2015), we show that the Ito's Lemma and the BS PDE remain valid when the Wiener process is extended to the $J$-process as a model for the underlying asset dynamics. Hence, if we call $\mathrm{V}$ the option price and $Q$ the rate of dividends of the underlying asset, then the BS related PDE operator, named $\mathrm{L}(\mathrm{V})$, is as follows:

$$
L(V)=\frac{\partial V}{\partial t}+\frac{1}{2} \sigma^{2} S_{t}^{2} \frac{\partial^{2} V}{\partial S^{2}}+(r-Q) S_{t} \frac{\partial V}{\partial S}-r V .
$$

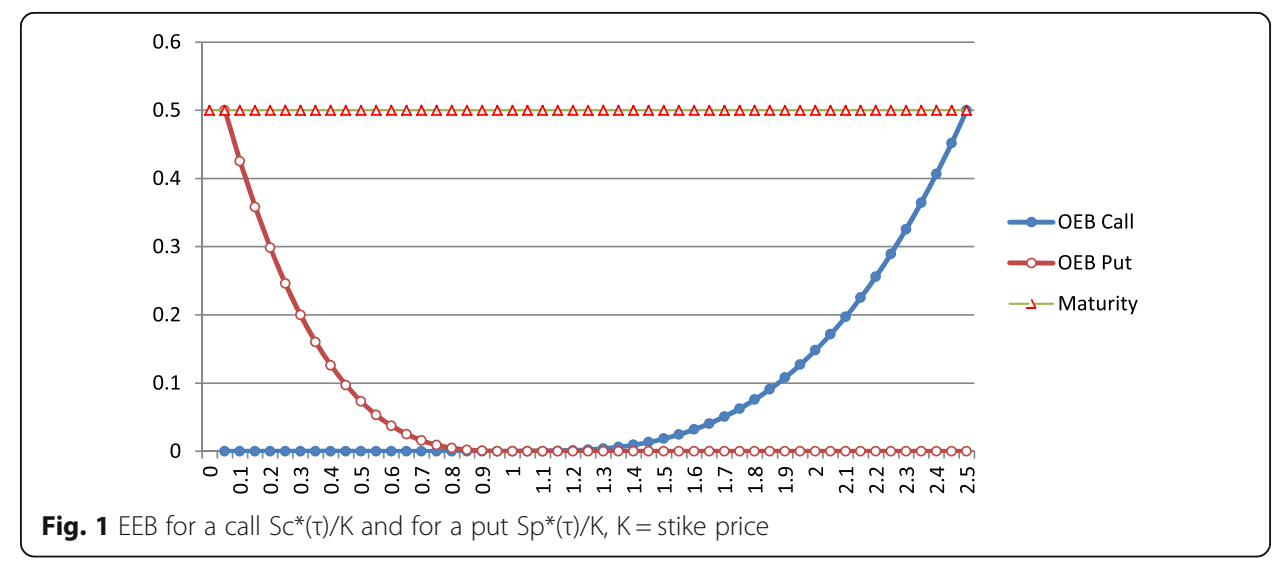


Hence, the European option price given by the $J$-formula is a solution of the PDE (1):

$$
L(V)=0
$$

The American option price $V_{J \text {-am }}$, generated by the $J$-am model under the $J$-process, is a solution of the PDE (2) (see Jerbi (2015) and Kwok (1998)):

$$
L(V)=f\left(S_{t}, \tau\right)
$$

where $f\left(S_{t}, \tau\right)$ denotes the cash flow function defined in Table 1 .

The American option is considered to be a compound option that includes a European option and an EEP. Then, the price $V_{J-a m}$ is the sum of two option prices: $V_{J-e u r}$ and $\varepsilon_{J} . V_{J-a m}=V_{J-e u r}+\varepsilon_{J}$, where $V_{J-\text { eur }}$ denotes the solution of the PDE (1), and $\varepsilon_{J}$ is a particular solution of the PDE (2). We denote the time to maturity by $\tau=T-t$ and the probability density of the underlying asset price at time $t$ by $\psi\left(S_{t}, t\right)$. From the Duhamel principle in the PDE theory (Zauderer 1989, page 2001), the general solution of the PDE (2) can be written as follows (please refer to the study by Kwok (1998)):

$$
\left\{\begin{array}{l}
V_{\mathrm{J}-\mathrm{am}}=V_{\mathrm{J}-\text { eur }}+\varepsilon_{J} \\
V_{\mathrm{J} \text { eur }}=e^{-r \tau} \int_{0}^{+\infty} V\left(S_{T}, T\right) \psi\left(S_{T}, 0\right) d S_{T} \\
\varepsilon_{J}=\int_{0}^{\tau} e^{-r \omega} \int_{0}^{+\infty} f\left(S_{\omega} ; \tau-\omega\right) \psi\left(S_{\omega}, \omega\right) d S_{\omega} d \omega .
\end{array}\right.
$$

When the American option is an American put, named $\mathrm{P}_{J-a m}$, the related boundary conditions are

C1: The option value at maturity $t=T$ is : $P_{J-a m}\left(S_{T}, T\right)=\operatorname{Max}\left(K-S_{T} ; 0\right)$,

C2: $S_{t}^{*}$ is the early exercise limit value at time $t$ belonging to the boundary OEB, such

as : $P_{J-a m}\left(S_{t}^{*}, t\right)=\operatorname{Max}\left(K-S_{t}^{*} ; 0\right)$,

C3: For $S_{t}=0$, we have $P_{J-a m}(0, t)=K$, and

C4:. $\left[\frac{\partial P_{I-a m}}{\partial S}\right]_{S=\infty}=0$

As the option is a put, in replacing the payoff and the function $\mathrm{f}$ by their own expressions, the system (3) becomes

$$
\left\{\begin{aligned}
P_{\mathrm{J} \text {-am }} & =P_{\mathrm{J} \text {-eur }}+\varepsilon_{J} \\
P_{\mathrm{J} \text {-eur }} & =e^{-r \tau} \int_{0^{+\infty}}\left(K-S_{T}\right)^{+} \psi\left(S_{T}, T\right) d S_{T} \\
\varepsilon_{J} & =\int_{0}^{\tau} e^{-r \omega} \int_{0}^{S_{(\tau-\omega)}}\left(r K-Q S_{\omega}\right) \psi\left(S_{\omega}, \omega\right) d S_{\omega} d \omega .
\end{aligned}\right.
$$

Under the $J$-process, the price $P_{J-\text { eur }}$ is computed using the $J$-formula found by Jerbi (2015), and the formula of $\varepsilon_{J}$ is detailed, in the Appendix.

The J-formula as a solution for European option pricing under J-process

As defined by Jerbi (2015), the closed form solution of PDE (1), i.e., the $J$-formula for a European call with underlying asset distributing dividends at a rate $Q$, is given by the 
following formula: $C_{t}=S_{t} e^{-Q \tau+\Phi}[1-F(X-b, \lambda, \theta+\lambda b)]-K e^{-r \tau}[1-F(X, \lambda, \theta)]$, where $F$ is the cumulative function of the statistical law, named $J$-law.

$$
\left\{\begin{array} { l } 
{ d _ { 2 } = \frac { \operatorname { L n } ( \frac { S _ { t } } { K } ) + ( r - Q + \frac { \sigma ^ { 2 } } { 2 } ) \tau } { \sigma = - d _ { 2 } \sigma _ { W } + E _ { W } } } \\
{ b = \frac { \sigma } { \sigma _ { W } } \sqrt { \tau } } \\
{ \Phi = \frac { b ^ { 2 } - \sigma ^ { 2 } \tau } { 2 } - b E _ { W } }
\end{array} \quad \left\{\begin{array}{l}
M(\lambda, \theta)=\frac{e^{-\frac{\theta^{2}}{2\left(1+\lambda^{2}\right)}}}{\operatorname{Jer}(\lambda, \theta) \sqrt{2 \pi\left(1+\lambda^{2}\right)}} \\
E_{W}=\lambda M(\lambda, \theta) \\
\sigma_{W}^{2}=1-\frac{\lambda^{2} \theta}{\left(1+\lambda^{2}\right)} M(\lambda, \theta)-\lambda^{2} M^{2}(\lambda, \theta)
\end{array}\right.\right.
$$

Applying the put- call parity formula, we deduce the formula of a put $P_{J-\text { eur }}$ :

$$
P_{J-e u r}=K e^{-r \tau} F(X, \lambda, \theta)-S_{t} e^{-Q \tau+\Phi} F(X-b, \lambda, \theta+\lambda b) .
$$

When the parameter $\lambda$ equals zero, the $J$-formula is exactly the BS one.

\section{The EEP value based on the J-formula}

For an American put, the value of the EEP equals to the sum of the actualized expected values of future cash flows between the date of the exercise and maturity. In the Appendix, we show that the value of the American EEP, under the $J$-process, equals

$$
\varepsilon_{J}=\int_{0}^{\tau} G(\omega) d \omega
$$

with $G(\omega)=\left\{r K e^{-r \omega} F\left(X_{(\tau-\omega)}^{*}, \lambda, \theta\right)-Q S_{t} e^{-Q \omega+\Phi} F\left(X_{(\tau-\omega)}^{*}-b, \lambda, \lambda b+\theta\right)\right\}$, where :

$$
\begin{aligned}
X_{(\tau-\omega)}^{*} & =-\sigma_{W} d_{2, \omega}+E_{W} \quad \text { and } \quad b=\frac{\sigma}{\sigma_{W}} \sqrt{\omega} \\
d_{2, \omega} & =\frac{\operatorname{Ln}\left(\frac{S_{t}}{S_{(\tau-\omega)}}\right)+\left(r-Q-\frac{\sigma^{2}}{2}\right) \omega}{\sigma \sqrt{\omega}} \text { and } d_{1, \omega}=d_{2, \omega}+\sigma \sqrt{\omega} . .
\end{aligned}
$$

Hence, when setting the parameter $\lambda=0$, we have the EEP, based on the BS model:

$$
\varepsilon_{B S}=\int_{0}^{\tau}\left[r K e^{-r \omega} N\left(-d_{2, \omega}\right)-Q S_{t} e^{-Q \omega} N\left(-d_{1, \omega}\right)\right] d \omega .
$$

\section{American option pricing model based on the Heston model as a bi-dimensional approach}

Heston (1993) considered the variance $v_{t}$ instead of the volatility $\sigma_{t}=\sqrt{v_{t}}$ as a second state variable. For the pricing of the European option, Heston (1993) adopted the Cox-Ingersoll-Ross (CIR) process as dynamics of the variance, with parameters $\kappa, \theta, \eta$ indicating the mean-reverting speed, long-term mean, and volatility's volatility factor, respectively. The processes $d z_{S, t}, d z_{v, t}$ are Brownian motions associated with the state variable movements $S$ and $v$, respectively, with a constant correlation factor equal to $\rho$. The dynamics of the underlying asset in a risk-neutral world is given by the following system: 


$$
\left\{\begin{array}{l}
\frac{d S_{t}}{S_{t}}=r d t+\sqrt{v_{t}} d z_{S, t} \\
d v_{t}=\kappa\left(\theta-v_{t}\right) d t+\eta \sqrt{v_{t}} d z_{v, t} \\
\rho\left(d S_{t} ; d v_{t}\right)=\rho
\end{array}\right.
$$

Considering a market risk price $\alpha$, the closed form solution he found is a solution of the Garman PDE (homogeneous PDE). When the underlying asset distributes dividends, we replace $S_{t}$ by $S_{t} e^{-Q(T-t)}$, and the Heston closed form solution, for an European call, can be written as follows:

$$
C_{t}=S_{t} e^{-Q(T-t)} \phi_{1}\left(S_{t} e^{-Q(T-t)}, v_{t}, K, \tau, \kappa, r, \theta, \eta, \rho, \alpha\right)-K e^{-r \tau} \phi_{2}\left(S_{t} e^{-Q(T-t)}, v_{t}, K, \tau, \kappa, r, \theta, \eta, \rho, \alpha\right) .
$$

The value of a European put can be deduced by the call- put parity. For the American option, the EEP price is a particular solution of the Garman (1976) PDE, with the function $f\left(S_{t}, t\right)$ as a second member instead of zero, as mentioned in Table 1. Drawing an analogy with the solution found for the $J$-model and BS model, the premium can be deduced by replacing $\tau=T-t$ by $\omega$ and $K$ by $S^{*}(\tau-\omega)$. Hence, for pricing an American call with underlying asset distributing dividends at a rate $Q$, we obtain the following formula:

$$
\begin{aligned}
& \varepsilon_{t}^{(c)}=\int_{0}^{\tau}\left\{Q S_{t} e^{-Q \omega} \phi_{1,(\tau-\omega)}^{(c)}-r K e^{-r \omega} \phi_{2,(\tau-\omega)}^{(c)}\right\} d \omega, \\
& \text { Where : }\left\{\begin{array}{l}
\phi_{1,(\tau-\omega)}^{(c)}=\phi_{1}\left(S_{t} e^{-Q \omega}, v_{t}, S_{(\tau-\omega)}^{*}, \omega, \kappa, \theta, \eta, \rho, \alpha\right) \\
\phi_{2,(\tau-\omega)}^{(c)}=\phi_{2}\left(S_{t} e^{-Q \omega}, v_{t}, S_{(\tau-\omega)}^{*}, \omega, \kappa, \theta, \eta, \rho, \alpha\right) .
\end{array}\right.
\end{aligned}
$$

For an American put, the EEP formula based on the Heston model as follows:

$$
\varepsilon_{\text {Heston }}^{(p)}=\int_{0}^{\tau}\left\{r K e^{-r \omega} \phi_{2,(\tau-\omega)}^{(p)}-Q S_{t} e^{-Q \omega} \phi_{1,(\tau-\omega)}^{(p)}\right\} d \omega \text { with }\left\{\begin{array}{l}
\phi_{1,(\tau-\omega)}^{(p)}=1-\phi_{1,(\tau-\omega)}^{(c)} \\
\phi_{2,(\tau-\omega)}^{(p)}=1-\phi_{2,(\tau-\omega)}^{(c)} .
\end{array}\right.
$$

Heston's (1993) formula used in this study considers the dividends rate distribution Q.

\section{Empirical studies}

\section{General methodology to determine the EEB and American option value}

To determine the American option price, we need the Snell envelop. This can be done step by step. We subdivide the time into intervals of length $\Delta \omega$. At each time, $\omega$, between the current time $t$ and maturity $T$, can be written as follows: $\omega=i \Delta \omega$ with $0 \leq i \leq N$ (Fig. 2). The first point of the EEB corresponds to $\omega=0$, i.e., $i=0$ corresponding to maturity $T$.

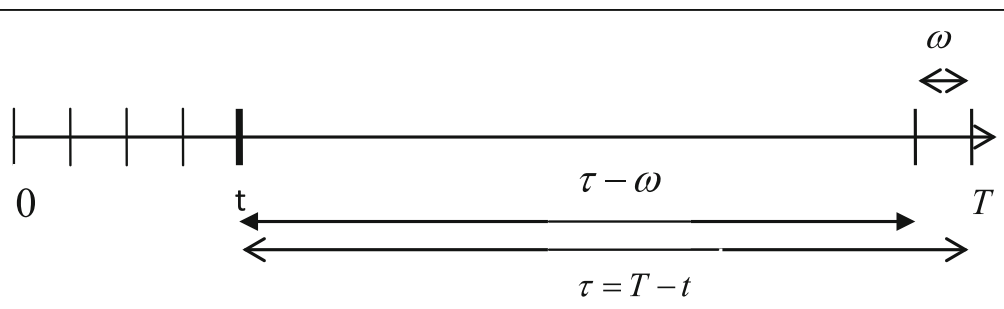

Fig. 2 Time to maturity for an American option 
At this time, the EEP $\varepsilon$ equals zero. Thus, the value of the American option equals that of the European option, which equals the intrinsic value of the option. Since $\varepsilon$ equals zero, we have $S^{*}(i=0)=\operatorname{Max}\left(K, \frac{r}{Q} K\right)$ for a call. For various values of $\mathrm{i}$, the points of the EEB $S_{(\tau-\omega)}^{*}$ are the solutions of the equation:

$$
S_{(\tau-\omega)}{ }^{*}-K=C_{e u r,(\tau-\omega)}+\varepsilon\left(S_{(\tau-\omega)}^{*}, \omega\right) \text { with } \omega=i \Delta \omega
$$

We use an iterative calculus to determine all the points $\left\{S_{(\tau-\omega)}^{*}, \omega=i \Delta \omega, 0 \leq i \leq N\right\}$ of the EEB. For a put, we have: $S^{*}(i=0)=\min \left(K, \frac{r}{Q} K\right)$, and the EEB can be determined by iteratively solving the equations:

$$
K-S_{(\tau-\omega)}^{*}=P_{e u r,(\tau-\omega)}+\varepsilon\left(S_{(\tau-\omega)}^{*}, \omega\right) .
$$

Regardless of the model chosen, $J$-am, BS-am, or Heston-am, to determine all the put EEB points $S^{*}(i)$, we propose the algorithm presented in Table 2.

After determining the EEB, we can determine the American option price at the current time $t$ in accordance with the formulas related to each of the three aforesaid models: BSam, $J$-am, and Heston-am, where BS-am constitutes a particular case of $J$-am when $\lambda$ equals to 0 . For further work, we will consider both $r$ and $Q$ to equal $5 \%$.

\section{Results and discussion}

Notations

Let $P_{B S-\text { eur }}\left(P_{B S-a m}\right)$ denote the price of a European put using the BS model (the price of the related American put using the BS-am model). Then, the related EEP represents

Table 2 Algorithm to determine the OEB for the BS-am, the J-am or the Heston-am model

Initialisation: For a put: $S^{*}(i=0)=\min \left(K, \frac{r}{Q} K\right)$

For $\mathrm{i}=1$ to $\mathrm{N}$

$\omega_{i}=i \Delta \omega$

$S e=S^{*}(i-1)$

$$
\begin{aligned}
& \text { Calcul } \operatorname{int}(i)=\sum_{p=1}^{p=i} G\left(S_{t}, S^{*}(i-p)\right) \Delta \omega \\
& \text { Calcul Peur }\left(\omega_{\mathrm{i}}\right) \\
& \quad S e_{0}=\operatorname{Se} \\
& \quad S e=K-(P e u r+\operatorname{int}(i)) \\
& \text { Until }\left|S e-S e_{0}\right|<\xi \\
& S^{*}(i)=S e: \text { OEB limit value related to time }\left(\tau-\omega_{i}\right)
\end{aligned}
$$

Next i 
Table 3 Notation of functions = price difference between the J-model and the Heston model. Notation of their minimas and maximas on the moneyness space

\begin{tabular}{|c|c|c|c|c|c|}
\hline \multicolumn{3}{|l|}{ J-model vs BS model } & \multicolumn{3}{|l|}{ Heston-model vs BS model } \\
\hline Function & Maxima & Minima & Function & Maxima & Minima \\
\hline$\Delta_{J-\text { eur }}=P_{J-\text { eur }}-P_{B S-\text { eur }}$ & $M_{j-\text { eur }}$ & $m_{\jmath-\text { eur }}$ & $\Delta_{\text {Heston- eur }}=P_{\text {Heston- eur }}-P_{B S-\text { eur }}$ & M Heston-eur & $m_{\text {Heston-eur }}$ \\
\hline$\Delta_{\jmath-a m}=P_{J-a m}-P_{B S-a m}$ & $M_{\text {J-am }}$ & $m_{\jmath-a m}$ & $\Delta_{\text {Heston-am }}=P_{\text {Heston-am }}-P_{B S-a m}$ & $M_{\text {Heston-am }}$ & $m_{\text {Heston - am }}$ \\
\hline$\varepsilon_{J}=P_{J-a m}-P_{J-\text { eur }}$ & $M_{\jmath}$ & $m_{\jmath}$ & $\varepsilon_{\text {Heston }}=P_{\text {Heston }-a m}-P_{\text {Heston }- \text { eur }}$ & $M_{\text {Heston }}$ & $m_{\text {Heston }}$ \\
\hline$\Delta_{\lrcorner-\varepsilon}=\varepsilon_{\jmath}-\varepsilon_{B S}$ & $M_{J-\varepsilon}$ & $m_{\jmath-\varepsilon}$ & $\Delta_{\text {Heston }-\varepsilon}=\varepsilon_{\text {Heston }}-\varepsilon_{B S}$ & $M_{\text {Heston- }-\varepsilon}$ & $m_{\text {Heston }-\varepsilon}$ \\
\hline
\end{tabular}

the difference: $\varepsilon_{B S}=P_{B S-a m}-P_{B S-\text { eur }}$. We denote by $P_{J-\text { eur }}\left(P_{J-a m}\right)$ the price of a European put using the $J$-formula (the price of the related American put using the $J$-am model). Moreover, we denote by $P_{\text {Heston-eur }}\left(P_{\text {Heston-am }}\right)$ the price of an European put using the Heston model (the price of the related American put using the American Heston-based model). Hence, either for the $J$-model or the Heston model, we can compute the following quantities with reference to the BS model (Table 3).

These aforementioned notations are used in the following empirical study.

\section{Comparison between the $\mathrm{J}$-am model (with $\lambda=0$ ) and the BS-am one}

In Figs. 3 and 4, we plot the EEB curves of the BS-am and $J$-am $(\lambda=0)$ models and their differences. We notice that the EEB curves conform with the theoretical ones plotted in Fig. 1 for an American put. In Fig. 3, we notice that the EEB curves for both models, BS-am and $J$-am $(\lambda=0)$, approximately coincide since their difference is located in an interval [-0.04; 0.05]. Indeed, this error corresponds to the threshold authorized by iterative calculations. With a shorter time step $\Delta \omega$ and with a longer computation time, this error can be reduced to an even lower level. For the two aforesaid models, we plot, in Fig. 5, both the American and European option values as a function of their moneyness. Moreover, we plot, in Fig. 6, their EEP value $\varepsilon$ as a function of moneyness. The curves in Figs. 5 and 6 are in accordance with the option theory and empirically confirm that the BS-am and $J$-am $(\lambda=0)$ models are the same. For all these computations, we

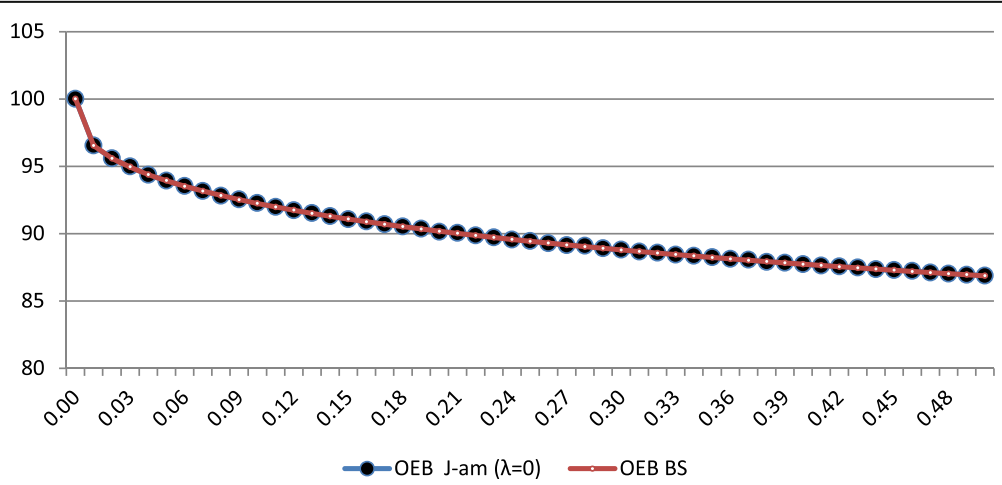

Fig. 3 Comparison of the EEB given by the models BS-am and $J$-am $(\lambda=0)$. Parameters values: $K=100$, $\tau=0.5, \sigma=10 \%, r=5 \%, Q=5 \%, \theta=0.769$ and $N=50$ 


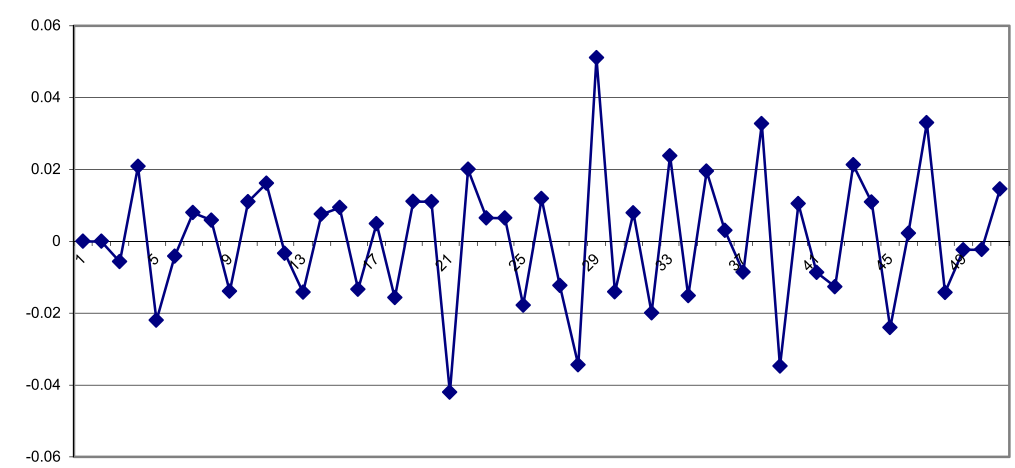

Fig. 4 Difference between $\mathrm{J}$-am $(\lambda=0)$ and BS-am models, in terms of EEB. Parameters values: $K=100$, $\tau=0.5, \sigma=10 \%, r=5 \%, Q=5 \%, \theta=0.769$ and $N=50$

consider a numerous time steps $N$ equal to 50. The CPU time required by the $J$ model to compute the American option value is relatively longer than the one needed by the $J$-am model. This is because of the absence of a W-function library, allowing a quick computation, while we have a library for the standard normal law cumulative function. We should build such a library to ensure a quasiinstantaneous calculus of the American option value. In this paper, for further calculations and to perform them in a reasonable time, we take $N=10$ instead of 50 or more. Although this choice slightly affects the accuracy of the results, it does not impact the results, curve profiles, and related conclusions.

\section{American pricing model results based on the $J$-am model}

To examine the effects of the parameters $\lambda$ and $\theta$ on the EEB curve profile and American option price related to $J$-am model, we plot, in Fig. 7 , the EEB curves for various values of $\lambda:(\lambda=1.6$ or $\lambda=-1.6)$ and $\theta:(\theta=-3, \theta=0$ or $\theta=3)$. We notice that the effect of $\lambda$ on the EEB profile is significant while the sensitivity to $\theta$ is very weak. The level of $S_{\tau}^{*}$, as a limit value to early exercise of the option at a given time, decreases with $\lambda$. For a given $\lambda$, the effect of $\theta$ (which ranges from -3 to +3 ) is not

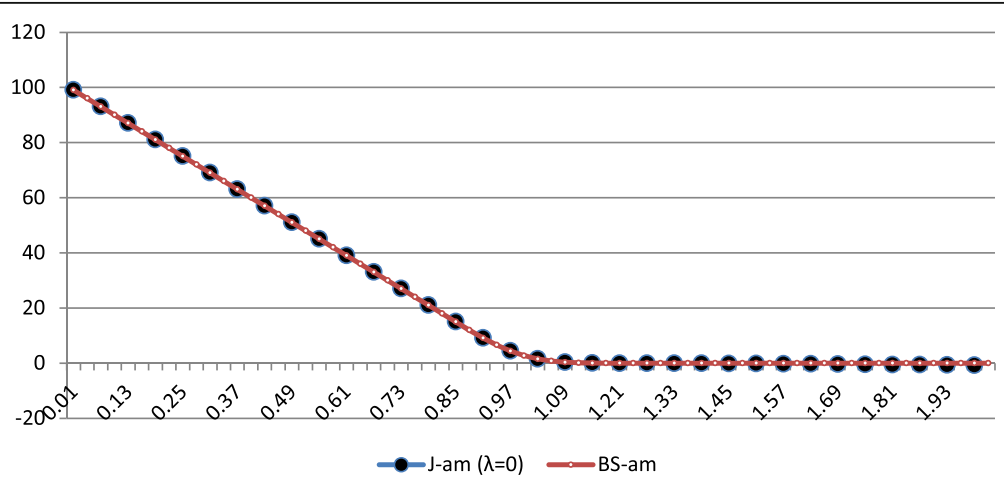

Fig. 5 Comparison between BS-am and $J$-am $(\lambda=0)$ models, in terms of European and American put prices. Parameters values: $\mathrm{K}=100, \tau=0.5, \sigma=10 \%, r=5 \%, Q=5 \%, \theta=0.769$ and $\mathrm{N}=50$ 


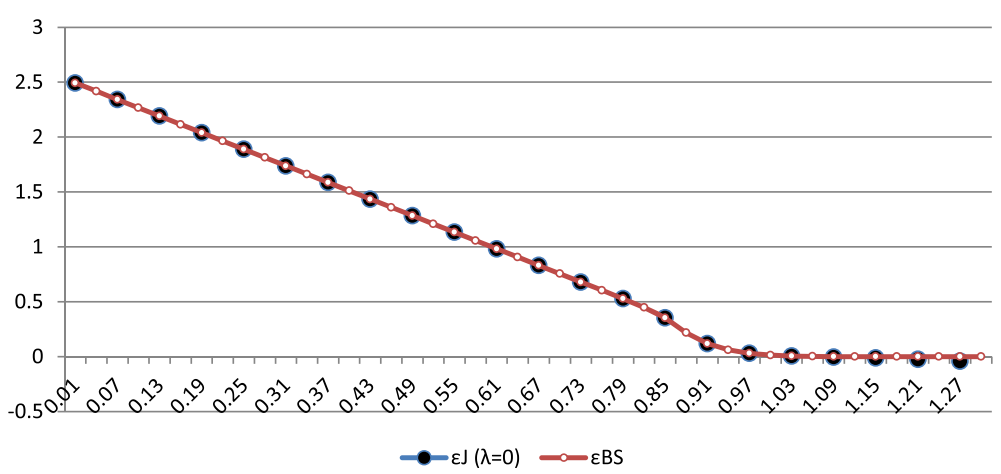

Fig. 6 Comparison between BS-am and J-am $(\lambda=0)$ models, in terms of American early exercise put price. Parameters values: $\mathrm{K}=100, \tau=0.5, \sigma=10 \%, r=5 \%, Q=5 \%, \theta=0.769$ and $N=50$

significant. For $\theta=0, S_{\tau}^{*}$ is maximum when $\lambda$ is negative and minimum when $\lambda$ is positive. The EEB curve related to the BS-am model is expected to be the same as the one related to $J$-am with $\lambda=0$, as plotted in Figs. 3 and 4. In Fig. 8, we represent the differences $\Delta_{J-\text { eur }}, \Delta_{J-a m}$, and $\Delta_{J-\varepsilon}$ related to the parameters values $(\lambda=$ -1.6 and $\theta=0)$. We notice that the curve $\Delta_{J-\text { eur }}$ is the same as the one elaborated by Jerbi (2015). For a put, regardless of the moneyness, the difference $\Delta_{J-\varepsilon}=\varepsilon_{J}$ $-\varepsilon_{B S}$ is positive. With reference to the BS model, for negative values of $\lambda$, the $J$ model overvalues the out-of-the-money European put and undervalues the in-themoney ones. With reference to the BS-am model, the $J$-am model overvalues the American puts when moneyness is less $\mu_{1}^{*}<1$ or greater than $\mu_{2}^{*}>1$, whereas it undervalues the American puts with moneyness located in the interval $\left[\mu_{1}^{*} ; \mu_{2}^{*}\right]$. The differences: $\Delta_{J-a m}=P_{J-a m}-P_{B S-a m}$ and $\Delta_{J-\varepsilon}=\varepsilon_{J}-\varepsilon_{B S}$ present positive maximums for a moneyness $\mu^{*}<\mu_{1}^{*}<1<\mu_{2}^{*}$. Besides, for positive values of $\lambda$, the profile of the curve $\Delta_{J-a m}$ is nearly the same as the one of $\Delta_{J-\text { eur }}$. The difference $\Delta_{J-\varepsilon}$ $=\varepsilon_{J}-\varepsilon_{B S}$ is positive for in-the-money puts, whereas it is negative for out-of-the-money puts. In Figs. 9 and 10 , for $\lambda=-1.6$ and $\lambda=1.6$, respectively, we plot $\Delta_{J-\text { eur }}$ for

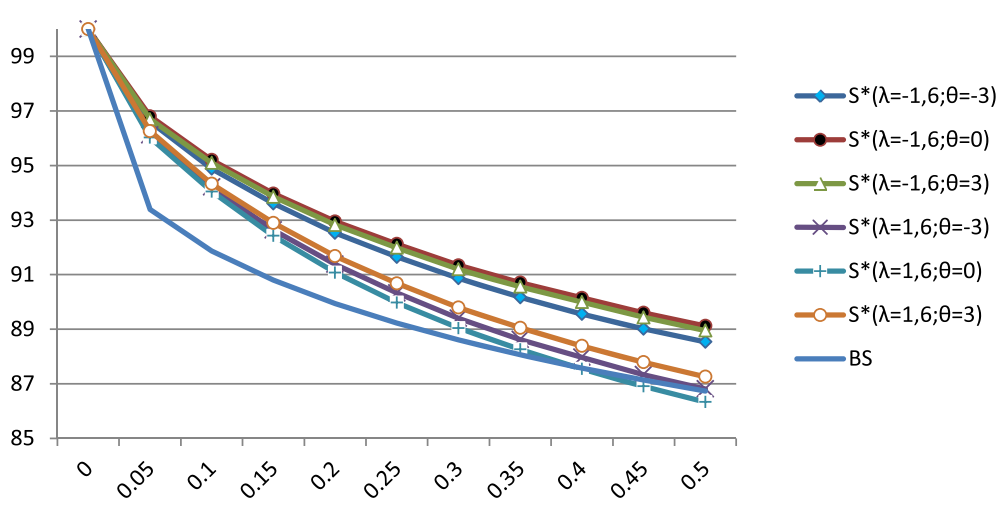

Fig. 7 EEB related to the BS-am and $J$-am for various values of the parameters $\lambda$ and $\theta$. Parameters values: $K=100, \tau=0.5, \sigma=10 \%, r=5 \%, Q=5 \%$ 


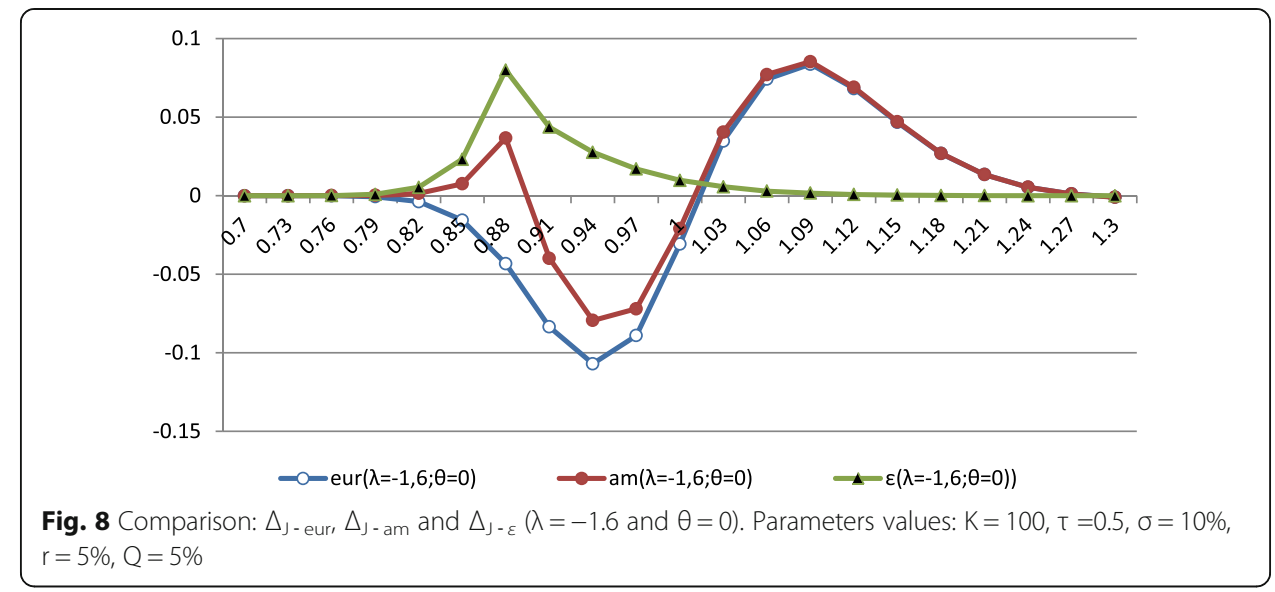

various values of $\theta$. We do the same for the differences $\Delta_{J-a m}$ (Figs. 11 and 12) and $\Delta_{J-\varepsilon}$ (Figs. 13 and 14).

Regarding $\Delta_{J-\text { eur }}$ we confirm the results found by Jerbi [1]. Regarding the value of $\lambda$, the maximum and the minimum of the difference $\Delta_{J-\text { eur }}$ are related to $\theta=0$. These extreme values are close to those related to $\theta=3$ and $\theta=-3$. Regarding $\Delta_{J}$ - am , for positive values of $\lambda$, the curve profile function of the moneyness is quite similar to the one related to $\Delta_{J-e u r}$. For negative values of $\lambda$, the curve profile is almost the same as the corresponding $\Delta_{J-\text { eun }}$ with the only difference that it presents a positive maximum located in the moneyness interval $[0.85 ; 0.9]$. This maximum coincides with the maximum of $\Delta_{J-\varepsilon}$, as indicated in Fig. 13. In this figure, we see that the effect of $\theta$ on $\Delta_{J-\varepsilon}$ is negligible when $\lambda$ is negative. However, when $\lambda$ is positive, the $\varepsilon_{J}$ value is sensitive to changes in the parameter $\theta$. This is because the $\varepsilon_{J}$ value increases with the absolute value of this parameter, with a maximum value corresponding to the same moneyness.

\section{Equivalence condition between the $J$-am model and Heston-am one}

The comparison between the $J$-am and Heston-am models is performed with reference to the BS and BS-am models. Hence, we examine the skewness and kurtosis effects on

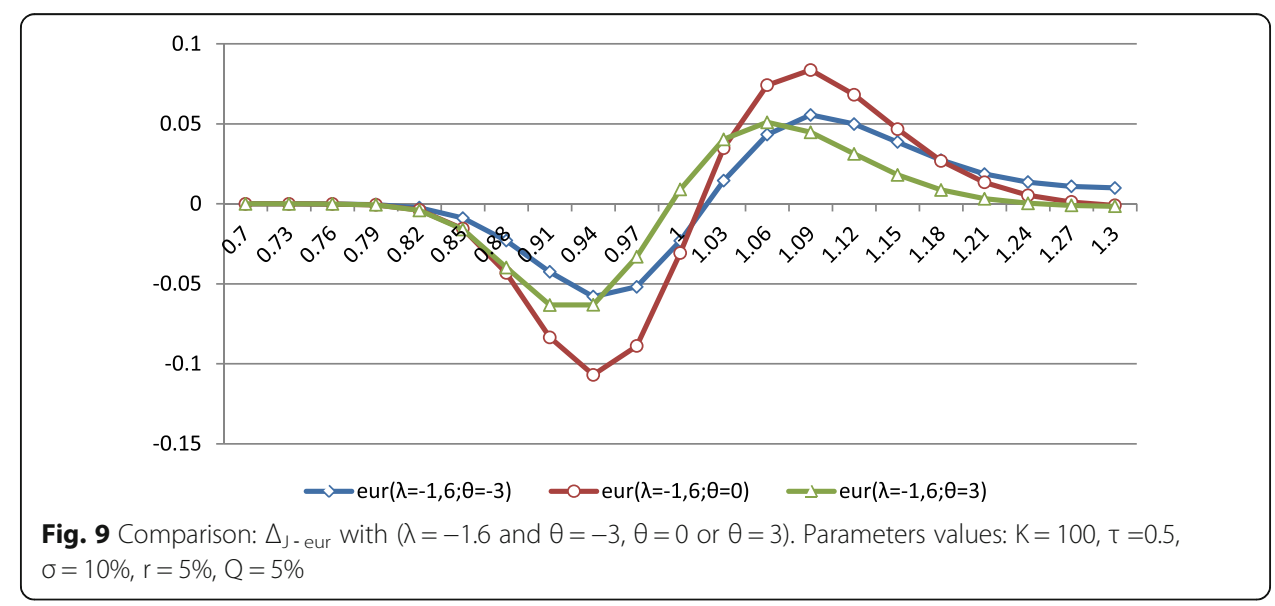




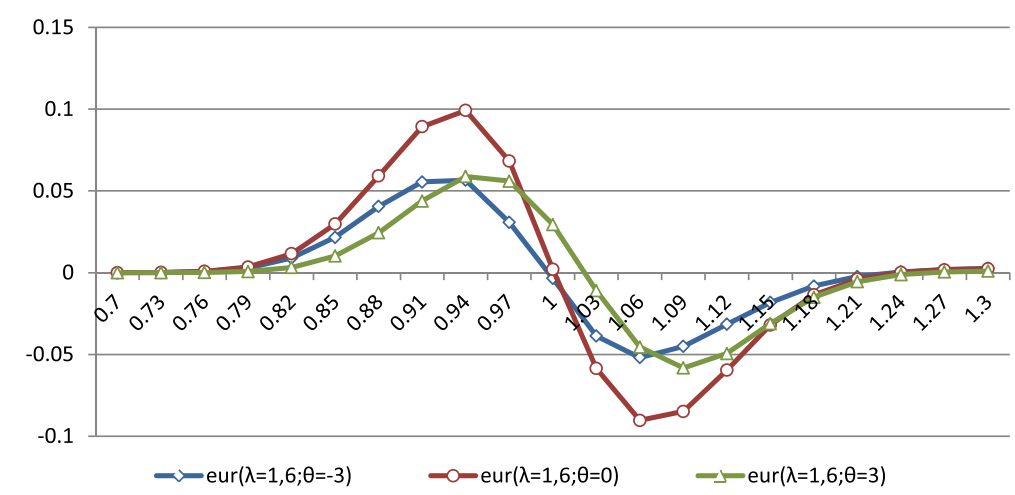

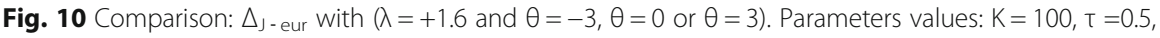
$\sigma=10 \%, r=5 \%, Q=5 \%$

American option pricing related to the two aforesaid models. As indicated by Jerbi (2015), we compare the J-model results with those of Heston (1993) with regard to European option pricing. We find that these two models give the same shapes of the curves $\Delta_{J-e u r}(S / E)$ for various values of the parameter $\rho(\lambda)$. To empirically compare the $J$-model and Heston one, we consider the same volatility parameter values used by Heston (1993) $(v=0.01 ; \sigma=0.1 ; \kappa=2 ; \phi=0.01$ and $\rho=0.5$ and -0.5$)$ and we plot the respective curves of option difference value with the one of the BS model. To determine the values of parameters $\lambda^{*}$ and $\theta^{*}$ regarding the $J$-model (see Jerbi (2015)) and those corresponding to the aforesaid Heston parameters, we use two approaches. In the first approach, equivalence is based on the dynamics of the underlying asset, in the second approach, the equivalence is based on the European option price $J$-formula and Heston's model. As indicated by Jerbi (2015), we find that $\lambda^{*}=0.84$ and $\theta^{*}=0.769$, with an estimation error equal to 6,782 E-07). In the second approach, to determine $\lambda^{*}$ and $\theta^{*}$ corresponding to the optimal equivalence, we minimize the gap between the $J$-model and Heston's model. For a given $\rho$, we estimate the values of $\lambda^{* \prime \prime}$ and $\theta^{* \prime \prime}$ by considering the minimum of the difference between the two model prices:

$$
\left(\lambda^{* *}, \theta^{* *}\right)=\operatorname{Arg} \min _{\lambda, \theta}\left\{J_{\text {eur }}(\lambda, \theta)-\text { Heston }_{\text {eur }}(\kappa, \phi, \eta, \rho)\right\}^{2}
$$

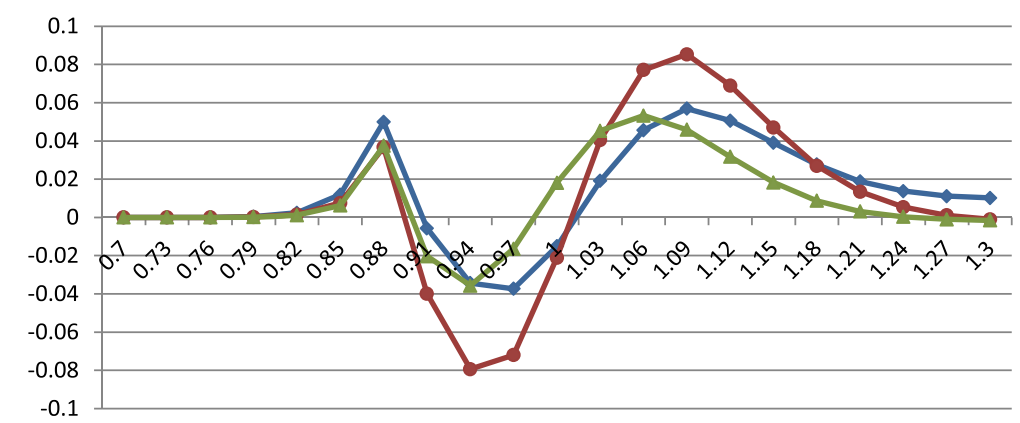

Fig. 11 Comparison: $\Delta_{\text {f } \text { am }}$ with $(\lambda=-1.6$ and $\theta=-3, \theta=0$ or $\theta=3)$. Parameters values: $K=100, \tau=0.5$, $\sigma=10 \%, r=5 \%, Q=5 \%$ 


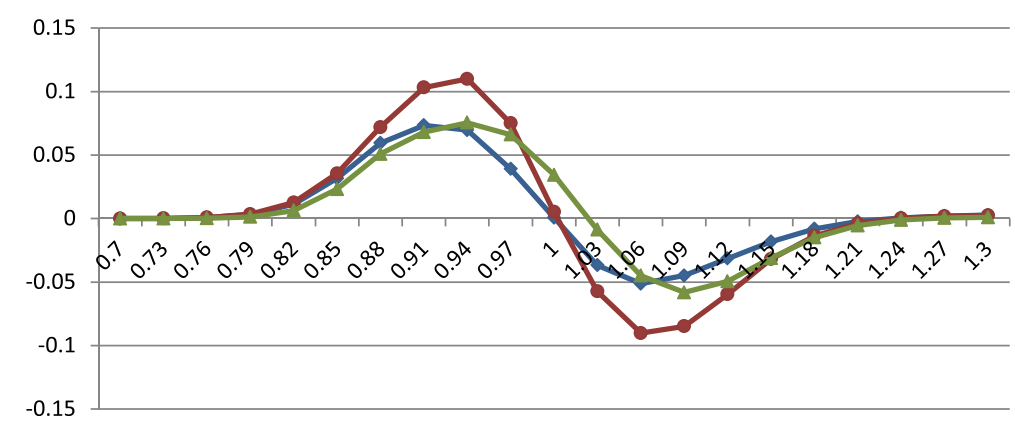

Fig. 12 Comparison: $\Delta_{\text {f } \text { am }}$ with $(\lambda=+1.6$ and $\theta=-3, \theta=0$ or $\theta=3)$. Parameters values: $K=100, \tau=0.5$, $\sigma=10 \%, r=5 \%, Q=5 \%$

We notice that using the second equivalence approach based on the volatility parameters used by Heston (1993) $(\nu ; \kappa ; \phi ; \eta$ and $\rho$ ), for $\rho=0.5$ and $\rho=-0.5$, the difference $\Delta_{\text {Heston-eur }}$ is almost at its minimum for $\lambda^{* \prime \prime}=1.6$ and $\lambda^{* \prime *}=-1.6$, respectively. Unlike the bi-dimensional Heston model, which is based on Fourier inversion, the $J$-model has the advantage of being a uni-dimensional model using a simple computational technique. We can say that "the use of only one state variable following the $J$ process" is equivalent to the use of two correlated state variables following the Wiener process. Here, we extend the equivalence to American options. If we consider the previous values of $\lambda=1.6$ and $\lambda=-1.6$ for the $J$-am model and $\rho=0.5$ and $\rho=-0.5$ for the Heston-am model, in plotting the respective EEB curves in Fig. 15, we find that the equivalence encountered in the study by Jerbi (2015), for the European options, remains true for the American options. These values of $\lambda$ are only approximate. Hence, they can be adjusted more accurately to fit the Heston model. In Fig. 12, we notice that regardless of the model chosen, the profile of the EEB curves fits the theory. For a given time, during the life of option, the early exercise is optimal for $S^{*}$. This level $S^{*}$ decreases with $\lambda$ for the $J$-am model and with $\rho$ for the Heston-am model. Moreover, we notice that the parameter value equivalence studied by Jerbi (2015) remains valid for the American option, since we have the correspondence $(\lambda=1.6$ and $\lambda=-1.6)$ in the $J$-am model to $(\rho=0.5$ and $\rho=-0.5)$ that in Heston's model. The EEB curve related to BS-am is considered to be the same as the $J$-am one with $\lambda=0$.

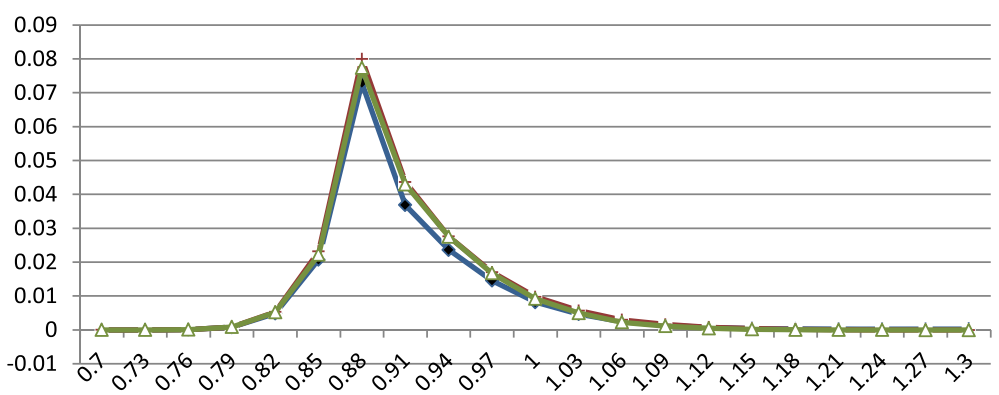

Fig. 13 Comparison: $\Delta_{\text {- } \varepsilon}$ with $(\lambda=-1.6$ and $\theta)$. Parameters values: $K=100, \tau=0.5, \sigma=10 \%, r=5 \%$ and $\mathrm{Q}=5 \%$ 


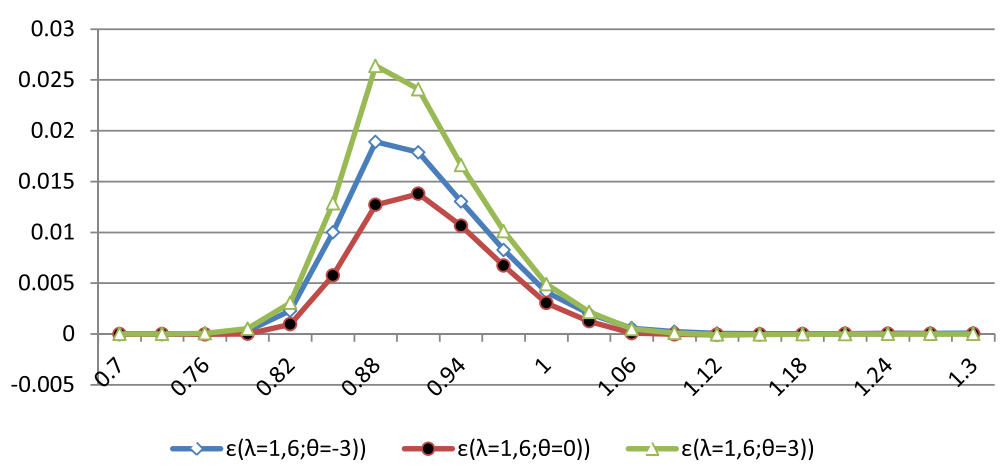

Fig. 14 Comparison: $\Delta_{\jmath}-\varepsilon$ with $(\lambda=+1.6$ and $\theta)$. Parameters values: $K=100, \tau=0.5, \sigma=10 \%, r=5 \%$ and $\mathrm{Q}=5 \%$

\section{Comparison between J-am model and Heston-am one in terms of the volatility effect when the equivalence condition is satisfied}

Since the option value is sensitive to the volatility change, we must examine the effect of such a variable on the option price for various values ranging from 10 to $50 \%$, with a rising gap of $10 \%$. We begin by examining the EEB curves of the $J$-am model with parameters $\lambda=-1.6$ and $\theta^{*}=0.769$, plotted in Fig. 16: we compare this with the Heston model related to the parameters $(\kappa=2 ; \phi=0.01$ and $\rho=-0.5)$ with a variance $v=0.01$, plotted in Fig. 17. We notice that although the curve profiles are quite similar, we find that only for $\sigma=10 \%$ (i.e. $v=0.01$ ), the $J$-am EEB exactly coincides with the Heston one. This is quite normal because for $\rho=-0.5$, the related value $\lambda=-1.6$ is based on the dynamics of the CIR used by Heston for a value of $v=0.01$. This means that for each value of $v$, we must compute the new related value of $\lambda$. Since we consider the same value of $\lambda$ regardless of the value of $v$, the equivalence between the two models, in terms of EEB, is valid only for $v=$ 0.01. The comparison between the J-model and Heston's model enables us to examine the skewness and kurtosis effects on the European or American put.

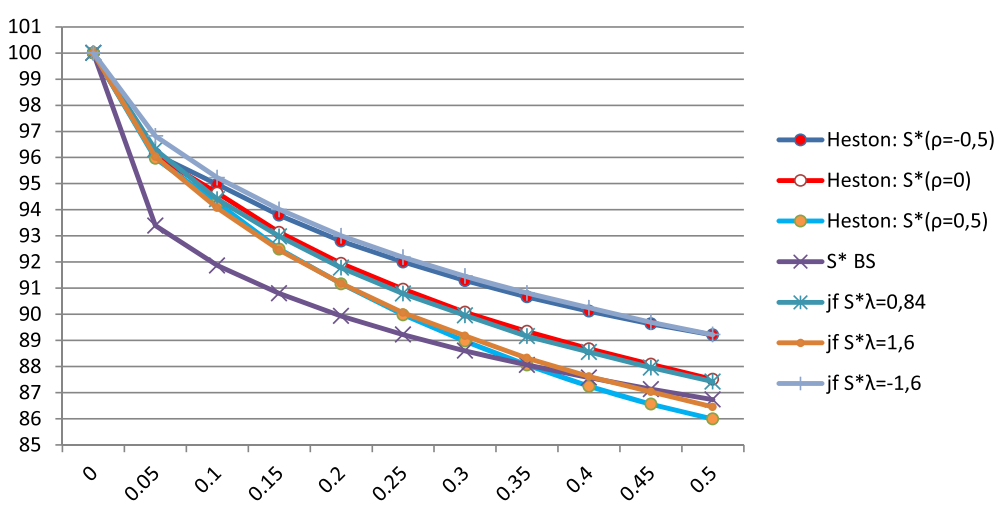

Fig. 15 Comparison of the EEB related to the BS-am and J-am and Hesston-am models, Parameters values: $K=100, \tau=0.5, \sigma=10 \%, r=5 \%, Q=5 \%$, J-am $(\lambda=0.84 ; 1.6$ and -1.6 with $\theta=0.769)$. Heston-am $(v=0.01$; $K=2 ; \varphi=0.01$ and $\rho=0.5 ; 0$ and 0.5 ) 


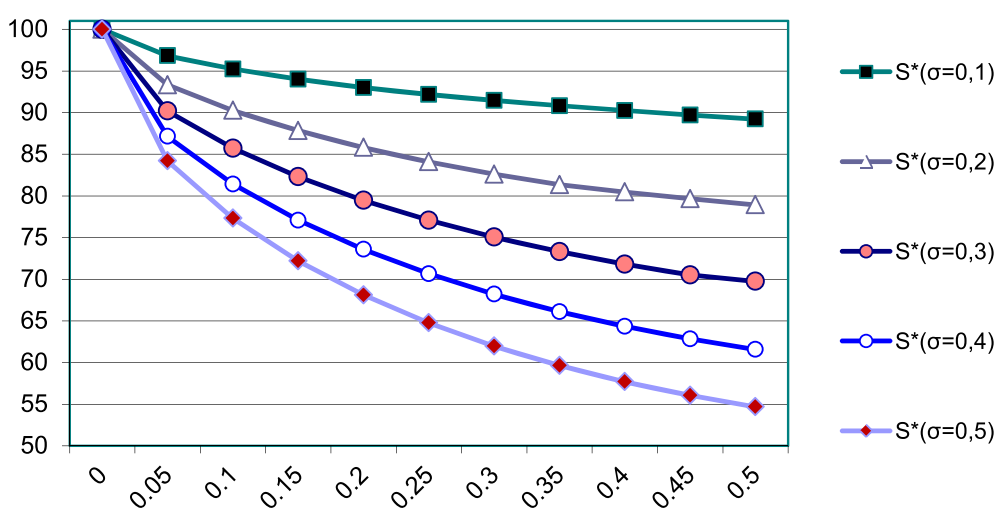

Fig. 16 EEB related to $\mathrm{J}$-am with parameters values: $K=100, \tau=0.5, \sigma=10 \%, r=5 \%, Q=5 \%$, J-am $(\lambda=-1.6$ with $\theta=0.769$ )

These effects are induced by the stochastic volatility effects in the Heston model and the $J$-process effects in the $J$-model. For $\lambda=-1.6$, we plot $\Delta_{J-a m}$ in Fig. 18: for the equivalent value $\rho=-0.5$, we plot $\Delta_{\text {Heston-am }}$ in Fig. 19. We notice that, for negative values of $\lambda$ and $\rho$, these two differences are negative and decrease with volatility. The value -1.6 of $\lambda$ was computed for $\rho=-0.5$ and for a variance $v=0.01$. If we adapt the estimation of the parameter $\lambda$ to the variance value, the $\Delta_{\text {Heston-am }}$ curves should be the same as the $\Delta_{J \text {-am }}$ ones plotted in Fig. 18. For the reason previously evoked, this is only the case for $\nu=0.01$. When $\lambda$ and $\rho$ are positive, $\Delta_{J-a m}$ and $\Delta_{\text {Heston-am }}$ are positive for in-the-money puts and negative for out-of-the-money puts. Hence, compared with the BS-am model, for negative values of $\lambda$ or $\rho$, (Figs. 18 and 19), the $J$-am and Heston-am models undervalue the American in-the-money puts and overvalue the out-of-the-money ones and vice versa. These curves are approximately the same for $(\lambda=-1.6$ and $\rho=-0.5)$ and for $(\lambda=1.6$ and $\rho=0.5)$, when the volatility equals $10 \%$. Besides, the curves related to $\varepsilon_{J}$ and $\varepsilon_{\text {Heston }}$ are similar, as shown in Figs. 20 and 21. The maximum of the premium increases with the volatility level and corresponds to a lower moneyness.

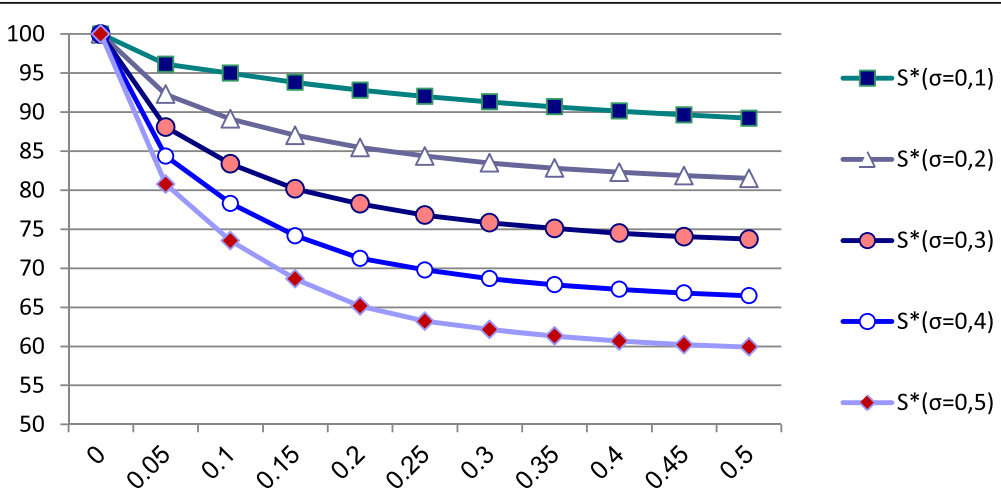

Fig. 17 EEB related to Heston-am models, with parameters values: $K=100, \tau=0.5, \sigma=10 \%, r=5 \%, Q=5 \%$, Heston-am ( $v=0.01 ; \mathrm{K}=2 ; \varphi=0.01$ and $\rho=-0.5)$ 


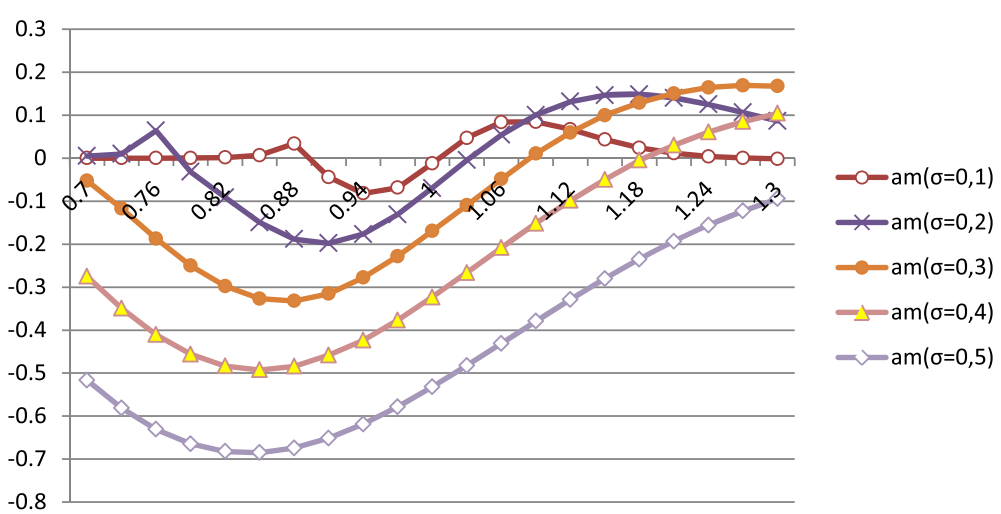

Fig. $18 \Delta_{\mathrm{J}-\mathrm{am}}$ as a function of moneyness, for various values of the Volatility, with parameters values: $K=100, \tau=0.5, \sigma=10 \%, r=5 \%, Q=5 \%,(\lambda=-1.6, \theta=0.769)$

\section{Comparison between J-am and Heston-am models in terms of the effect of the time to maturity}

Here, we examine the effect of the time to maturity (ranging from 0.1 to 1 year) on the American option price. For the European options, the problem is examined in the study by Jerbi (2015) and extended here to the American options. First, the EEB does not depend on the time to maturity, which means that for the values considered, we have the same curve support limited in time by the aforesaid values. We plot, in Fig. 22, the EEB related to the $J$-am model for various values of $\lambda$ as well as for the ones related to the Heston-am model for various values of the correlation factor $\rho$. The volatility is set equal to $10 \%$ for all the curves considered. The EEB $(\lambda=1.6)$ coincides with the one related to $(\rho=0.5)$, while the EEB $(\lambda=-1.6)$ coincides with the one related to $(\rho=-0.5)$. The EEB corresponding to the BS-am model is close to the Heston-am related to $(\rho=0)$. The precision of the price mainly depends on the accuracy of the determination of the EEB. Here, we have a tradeoff between the accuracy and the CPU time convergence. For a given time step, the EEB boundary does not depend on $\tau$ (Fig. 23).

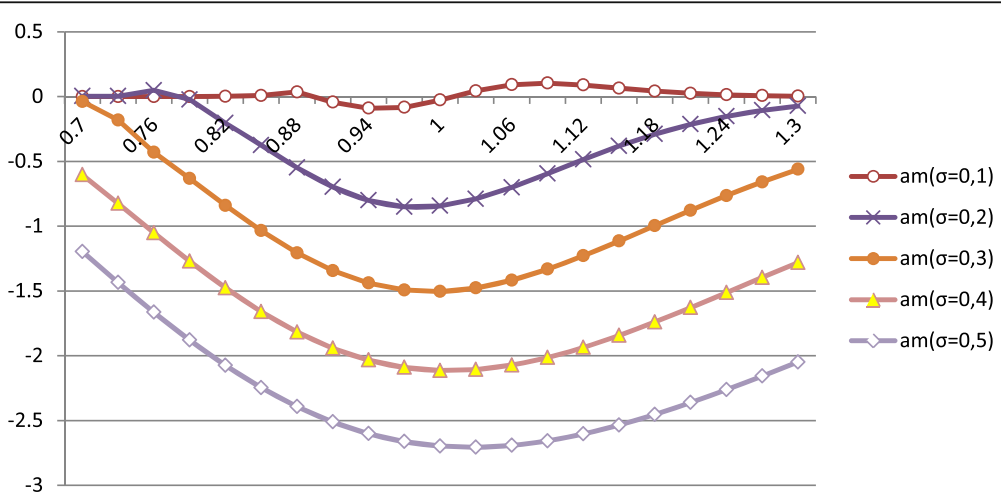

Fig. $19 \Delta_{\text {Heston - am }}$ as a function of moneyness, for various values of the volatility, with parameters values: $K=100, \tau=0.5, \sigma=10 \%, r=5 \%, Q=5 \%,(\lambda=-1.6, \theta=0.769) .(v=0.01 ; K=2 ; \varphi=0.01$ and $\rho=-0.5)$ 


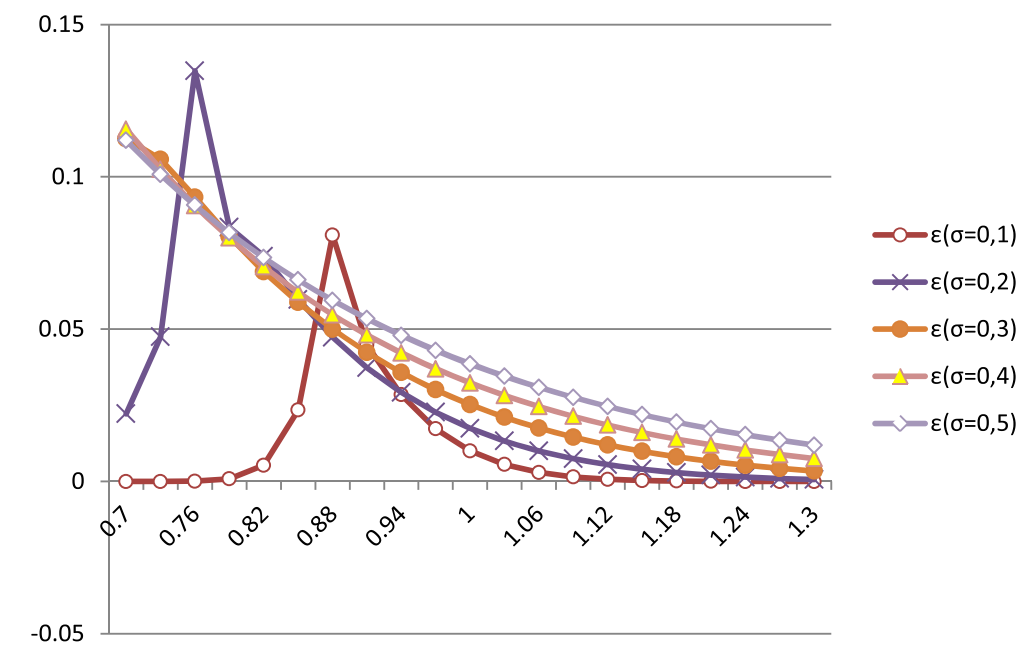

Fig. $20 \Delta_{\jmath}-\varepsilon$ as a function of moneyness for various values of volatility, with parameters values: $K=100$, $\tau=0.5, \sigma=10 \%, r=5 \%, Q=5 \%$, and $(\lambda=-1.6$ and $\theta=0.769)$

Unlike with the European puts, in the case of in-the-money American puts, when $\lambda$ and $\rho$ are negative, there is a small moneyness interval where the models $J$-am or Heston-am overvalue the option price compared with the BS-am model (Figs. 24 and 25). This interval does not exist for positive values of the parameters $\lambda$ and $\rho$ (Figs. 26 and 27). In all the other cases, the results for the American options are the same as those of the European ones encountered in the study by Jerbi (2015). Either for J-am model or the Heston-am one, the greater the time to maturity, the greater the difference with BS-am. Thus, we consider the EEP $\varepsilon_{J}$ and $\varepsilon_{H e s t o n}$ as functions of moneyness for the various values of the option maturity. Indeed, we examine the skewness and kurtosis effects on this option price. We notice that the two studied models give the same results for $\lambda=-1.6$ and $\rho=-0.5$ (Figs. 28 and 29), with positive values regardless of the moneyness. For each time to maturity $\tau$, $\varepsilon_{J}$ presents a maximum $\varepsilon\left(x_{\tau}^{*}\right)$ for a moneyness $x_{\tau}^{*}$ less than the unity. The value of $x_{\tau}^{*}$ decreases with $\tau$, while $\varepsilon\left(x_{\tau}^{*}\right)$

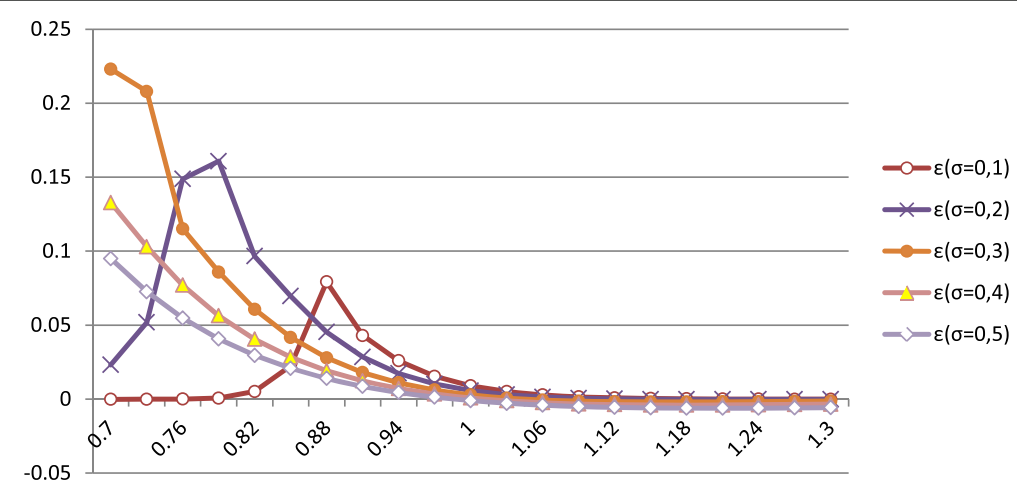

Fig. $21 \Delta_{\text {Heston }-\varepsilon}$ as a function of moneyness for various values of volatility, with parameters values: $K=100, \tau=0.5, r=5 \%, Q=5 \%$, and $(v=0.01, K=2, \varphi=0.01$ and $\rho=-0.5)$ 


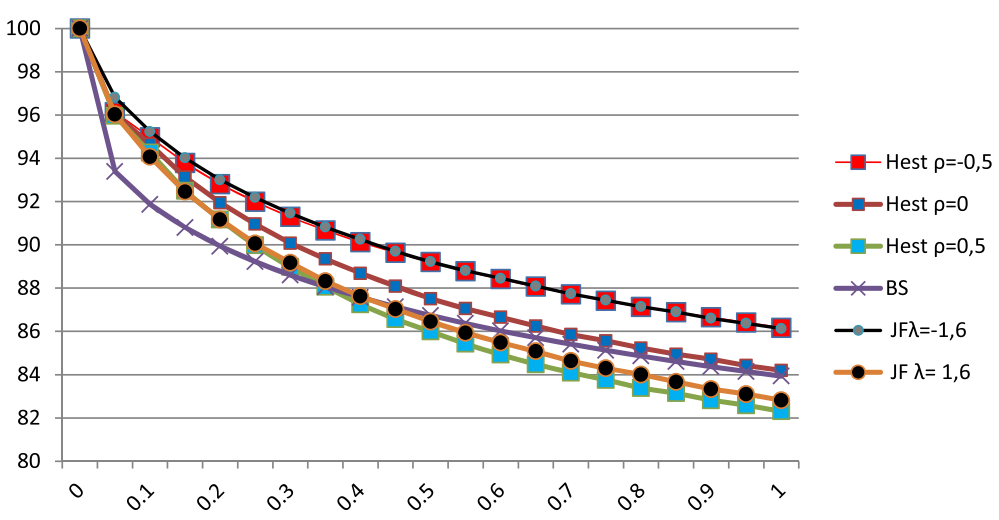

Fig. 22 Comparison between the EEB related to the BS-am and $J$-am and Heston- am models, Parameters values: $K=100, \tau=1, \sigma=10 \%, r=5 \%, Q=5 \%, J$-am $(\lambda=1.6$ and -1.6 with $\theta=0.769)$. Heston-am $(v=0.01$; $\mathrm{K}=2 ; \varphi=0.01$ and $\rho=0.5 ; 0$ and -0.5 )

increases. In case $\lambda=1.6$ and $\rho=0.5$, the $J$-am and Heston-am models give the same results. For $\tau$ less than almost $\tau^{*}=0.6$, the skewness and kurtosis induce positive values in the EEP, while for $\tau$ greater than $\tau^{*}$, we find a negative effect for a moneyness ranging from $x_{1, T}^{*}$ and a positive effect for a moneyness greater than $x_{1, \tau}^{*}$ (Fig. 30).

Finally, we can say that the comparison between the models is based on the effect of the stochastic volatility of the American option pricing. We have proved that the $J$-am is an extension of the model BS-am, as we did with the $J$-model and BS models. Furthermore, we conclude that the $J$-am model gives the same results as the Heston-am one in considering equivalent parameters ensuring equivalent effects of skewness and kurtosis. The equivalence between the two models can be improved with an accurate estimation of parameters $\lambda$ and $\theta$ for given values of the volatility dynamics parameters considered in the Heston model.

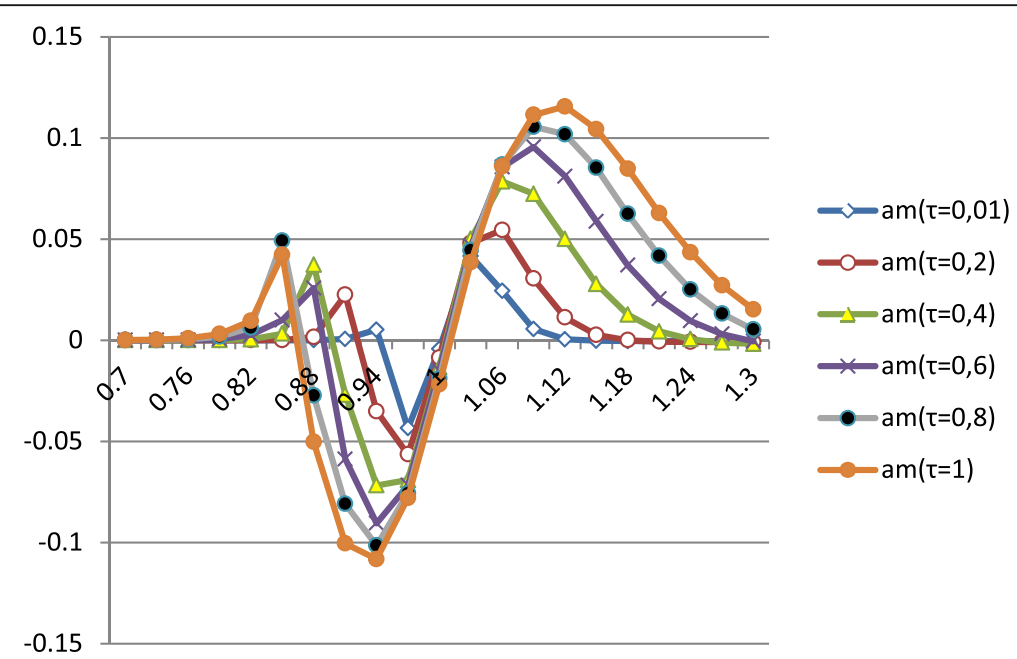

Fig. $23 \Delta_{\jmath}$ - am as a function of moneyness, for various values of time to maturity, Parameters values: $K=100, \tau=0.5, \sigma=10 \%, r=5 \%, Q=5 \%$, J-am $(\lambda=-1.6$ and $\theta=0.769)$ 


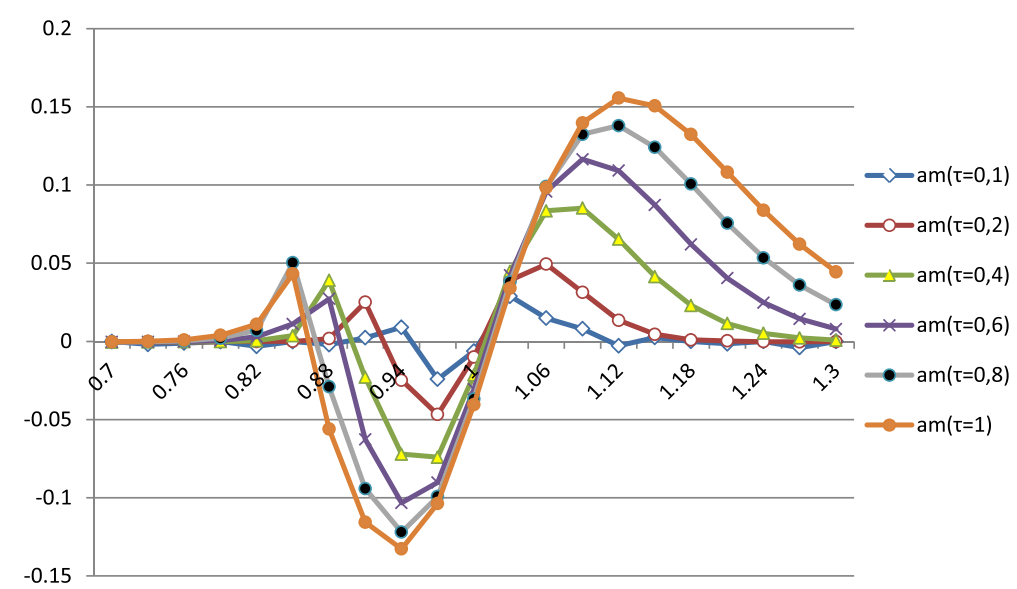

Fig. $24 \Delta_{\text {Heston - am }}$ as a function of moneyness, for various values of time to maturity. Parameters values: $K=100, \tau=0.5, r=5 \%, Q=5 \%,(v=0.01, K=2, \varphi=0.01$ and $\rho=-0.5)$

\section{Comparison between the $\mathrm{J}$-am and the Heston-am models in terms of CPU time}

For the three models, BS-am, Heston-am, and J-am, the CPU time required for computing the American put price is indicated in Table 4. The first part of this time CPU1 determines the EEB through 10 steps of the residual option lifetime. Once the 10 EEB points are computed, we calculate the American option value related to 21 levels of moneyness. This calculation requires the second part of time CPU2 such that CPU $=$ CPU1 + CPU2. The BS-am requires a relatively short CPU time, either for determining the EEB or for computing the American put value. The $J$-am model requires a long time to perform the same tasks. This is due to the fact that the basic function $W$ is numerically computed as an integral, which is time consuming. The Heston model, based on the Fourier inversion, requires a significantly lower CPU time than the one needed by the $J$-am model to compute the American put value. To drastically improve the speed of the $J$-am model convergence, we should build a library of the function $\mathrm{W}$, similar to the one corresponding to the standard normal cumulative function. Hence, the CPU time required by the $J$-am model will be the same as the one required by BS-am model and significantly better than the one required by Heston-am model. Since the $J$-am model considers the skewness and kurtosis effects, which is not the case with the BS-am model, the $J$-am represents the best

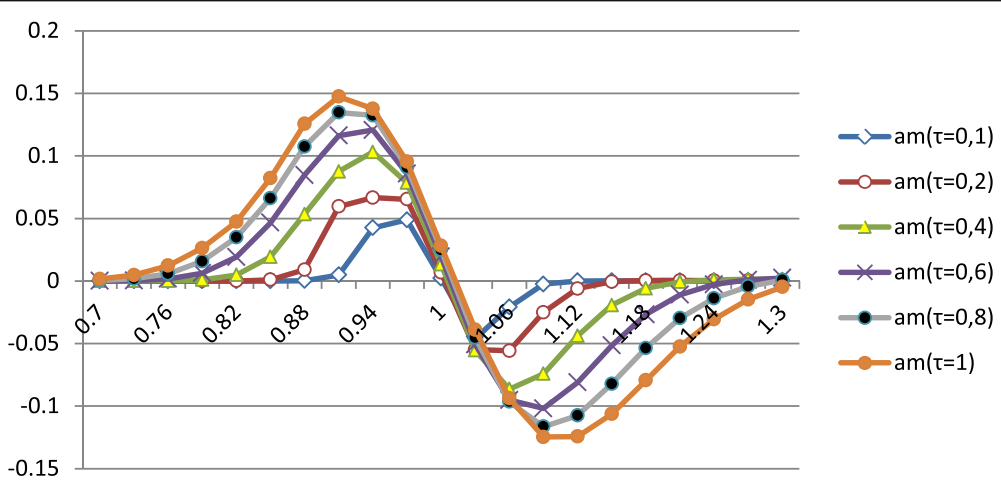

Fig. $25 \Delta_{\jmath}$ - am as a function of moneyness, for various values of time to maturity, Parameters values: $K=100, \tau=0.5, \sigma=10 \%, r=5 \%, Q=5 \%$, with $(\lambda=1.6$ and $\theta=0.769)$ 


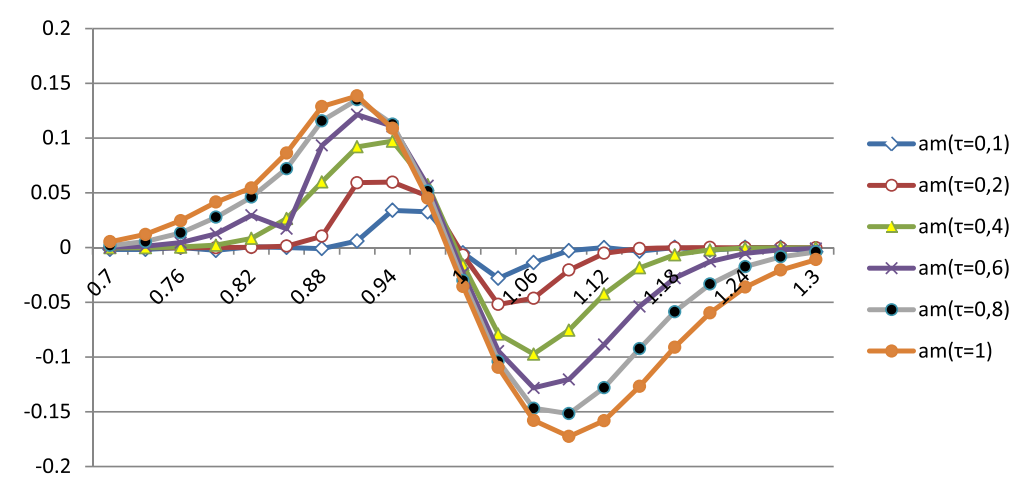

Fig. $26 \triangle_{\text {Heston - am }}$ as a function of moneyness, for various values of time to maturity. Parameters values: $K=100, \tau=0.5, r=5 \%, Q=5 \%$ with $(v=0.01, K=2, \varphi=0.01$ and $\rho=0.5)$

compromise between accuracy that conforms to the financial market reality and CPU time consumption.

\section{Improvements of the $J$-am model}

The valuation of the American option accuracy highly depends on the accuracy of the determination of the EEB. The errors are cumulated along the residual lifetime of the option. Hence, approaching the border with a high polynomial degree can preserve the accuracy of the calculation of this boundary. Hence, we can maintain the accuracy of the American option value. Simultaneously, we significantly reduce the required CPU time to compute the American option value. To do this, for the three models and reduce the computation complexity of the American option price, we model the EEB as a polynomial of a degree 10 (as we used 10 steps to determine the EEB) as follows:

$$
S_{\left(\tau-\omega_{i}\right)}^{*}=\sum_{k=0}^{k=10} \alpha_{k}\left(\tau-\omega_{i}\right)^{k}
$$

where $0 \leq \omega_{i} \leq \tau$ and is an integer : $0 \leq i \leq 10$.

If we denote by $\omega_{1}=\tau>\omega_{2}>\omega_{3}>\ldots \ldots \ldots . . .>\omega_{9}>\omega_{10}>0$ the values related to $\omega_{i}$ (with $0 \leq i \leq 10)$ and by $S^{*}{ }_{\left(\tau-\omega_{i}\right)}$ for the corresponding 10 limit values, which are computed

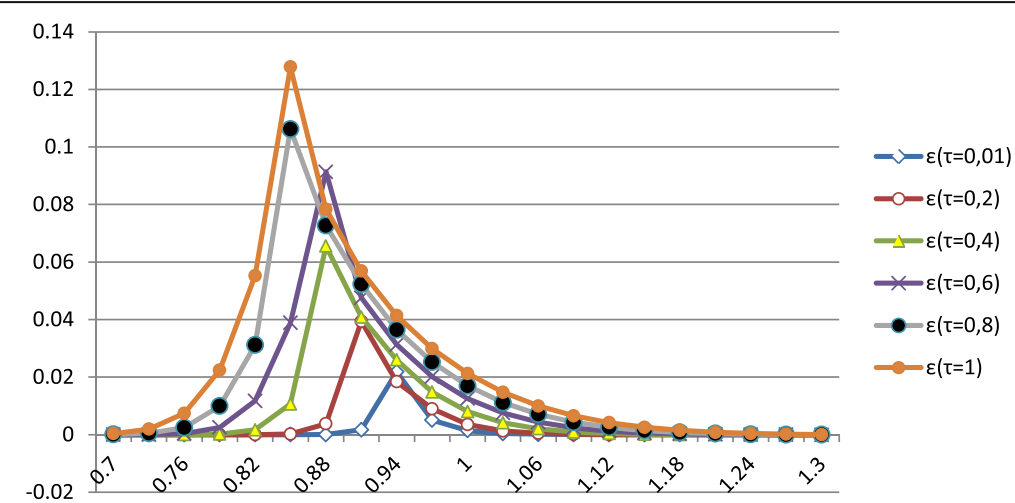

Fig. $27 \Delta_{\jmath-\varepsilon}$ as a function of moneyness for various values of time to maturity, Parameters values: $K=100$, $\tau=0.5, \sigma=10 \%, r=5 \%, Q=5 \%, J$-am $(\lambda=-1.6$ and $\theta=0.769)$ 


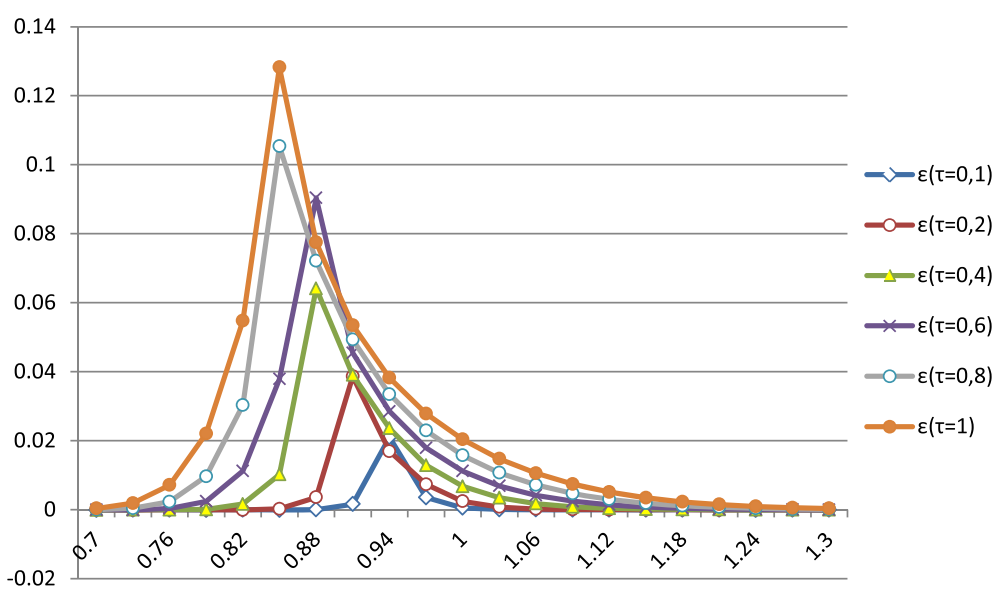

Fig. $28 \Delta_{\text {Heston }-\varepsilon}$ as a function of moneyness, for various values of time to maturity, Parameters values: $K=100, \tau=0.5, r=5 \%, Q=5 \%,(v=0.01, K=2, \varphi=0.01$ and $\rho=-0.5)$

with the aforesaid algorithm, we can determine the coefficients $\alpha_{k}$ (with $0 \leq k \leq 10$ ) of the polynomials through a Cramer system of degree 10. The coefficients related to Figs. 16 (J-am model) and 17 (Heston model) are given in Table 5. These coefficients are computed numerically. Therefore, we can express them as functions of the American option inputs to analytically compute the American option value.

\section{Conclusion}

In this study, we have elaborated a new uni-dimensional model $J$-am for pricing American options, which is based on the J-model developed by Jerbi (2015). We have shown that this model is an extension of the American model BS-am based on the BS model. As indicated by Jerbi (2015), we have examined the equivalence between the $J$-model and Heston one. Here, we have extended the study of this equivalence to American options. The parameters of the $J$-process ensuring this equivalence were determined as the values minimizing the squared errors between the $J$-process and CIR process used in the study by Heston (1993). We notice that these parameters can also be determined as the values minimizing the error between the $J$-am formula and Heston-am one. This study aimed to

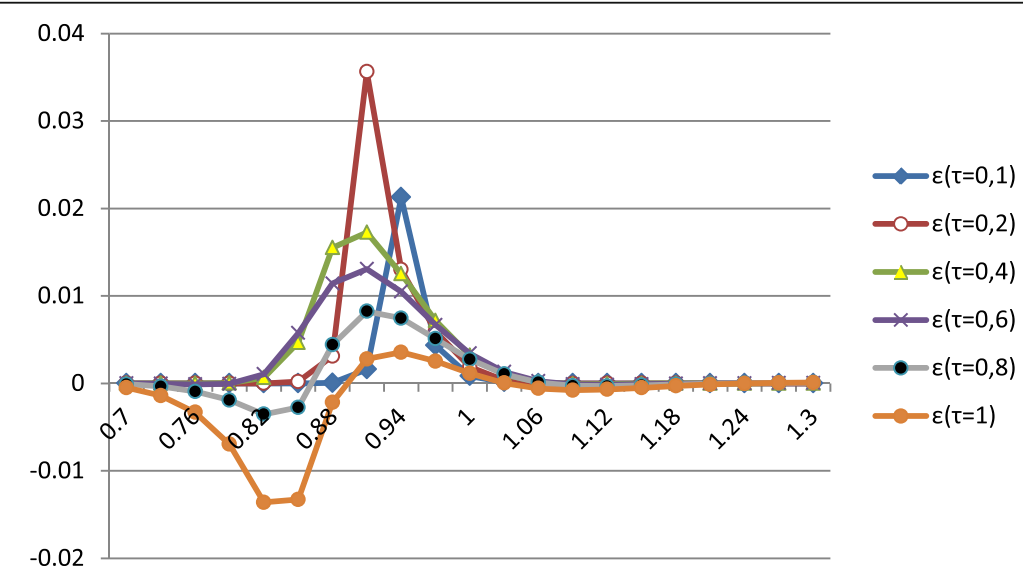

Fig. $29 \Delta_{\mathrm{J}-\varepsilon}$ as a function of moneyness for various values of time to maturity, with parameters values: $K=100, \tau=0.5, \sigma=10 \%, r=5 \%, Q=5 \%,(\lambda=1.6$ and $\theta=0.769)$ 


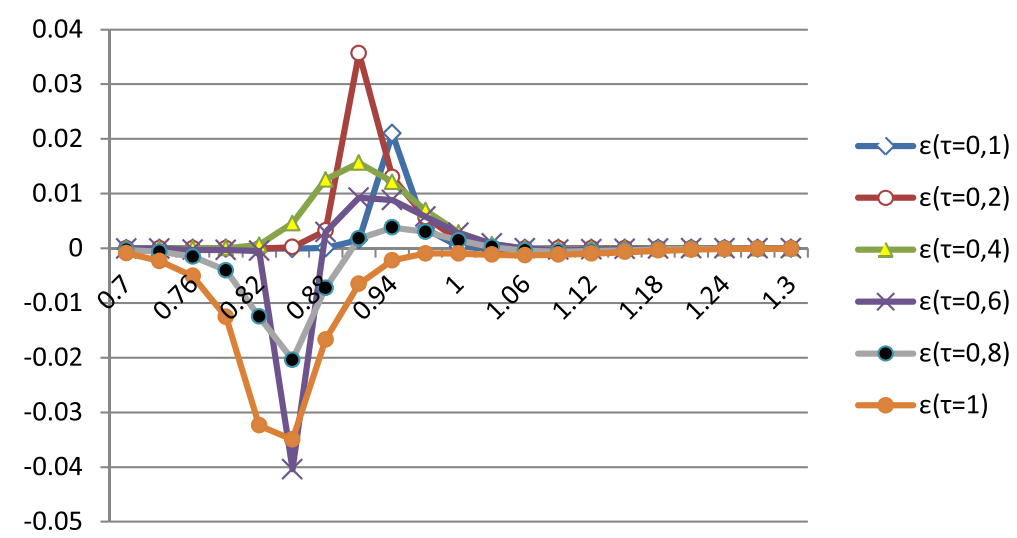

Fig. $30 \Delta_{\text {Heston - } \varepsilon}$ as a function of moneyness, for various values of time to maturity, with parameters values: $K=100, \tau=0.5, r=5 \%, Q=5 \%$, Heston-am $(v=0.01, K=2, \varphi=0.01$ and $\rho=0.5)$

compare a confirmed model, the Heston's, which is bidimensional, with an equivalent unidimensional model $J$-am. Assuming that we use the equivalent parameters $\lambda^{*}$ and $\theta^{*}$, we can say that our results regarding the $J$-am are totally in accordance with those of Heston's in terms of EEB and American option pricing. The EEB and the American option price profiles generated by all the chosen models fully conform with the options theory. We have examined the similarity between the effect of $\lambda^{*}$ and $\rho$ and the one between $\theta^{*}$ and $(\kappa ; \phi ; \eta ; v)$. We can say that the skewness and kurtosis effects induced by the stochastic aspect of the volatility in the bidimensional model of Heston, are equivalent to the ones generated by the extension of the Wiener process to the $J$-process. This was our conclusion, as indicated by Jerbi (2015), and we extended this conclusion to the American options. For a future work, we plan to examine the dynamic risk management related to an American option portfolio based on this model. We can also use this model to solve financial or economic problems based on American options, such as the decision optimization in an area characterized by innovation and technical progress. The $J$-am, as a uni-dimensional model, is expected to fit the reality of the financial market with a better compromise between accuracy and CPU time than the Heston-am model. The computation, based on the cumulative function $\mathrm{F}$ of the $J$-law, is easier than the one based on the Fourier inversion method used by Heston. A library for the function $\mathrm{F}$ must be constructed to ensure the optimality of the $J$-am model in terms of accuracy and time consumption. Moreover, the modeling of the EEB based on the polynomial approach can be carried out to significantly improve the CPU time needed to compute the American option value for a given accuracy. Finally, the results generated by the $J$-am model must be compared with those generated by simulations based on Malliavin calculus and using the $J$-process (see Jerbi and Kharrat (2014)).

Table 4 The CPU Time required at each stage for determining the American option

\begin{tabular}{llll}
\hline Time CPU (in second) & BS-am & Heston-am & J-am \\
\hline CPU1: OEB determination (10 points) & 1 & 430 & 5584 \\
CPU2: American Put value computing 21 points & 0 & 66 & 918 \\
\hline
\end{tabular}


Table 5 The OEB approximate polynomial coefficients for the $J$-am $(\lambda=-1.6, \theta=0.767)$ and Heston $(v=0.01, k=2, \varphi=0.01$ and $\rho=-0.5)$ models, for various values of the volatility

\begin{tabular}{|c|c|c|c|c|c|c|c|c|c|c|}
\hline \multirow[t]{2}{*}{ OEB } & \multicolumn{2}{|l|}{$S^{*}(\sigma=0,1)$} & \multicolumn{2}{|l|}{$\mathrm{S}^{*}(\sigma=0,2)$} & \multicolumn{2}{|l|}{$\mathrm{S}^{*}(\sigma=0,3)$} & \multicolumn{2}{|l|}{$\mathrm{S}^{*}(\sigma=0,4)$} & \multicolumn{2}{|l|}{$\mathrm{S}^{*}(\sigma=0,5)$} \\
\hline & J-am & Heston-am & J-am & Heston-am & J-am & Heston-am & J-am & Heston-am & J-am & Heston-am \\
\hline$a_{0}$ & 100 & 100 & 100 & 100 & 100 & 100 & 100 & 100 & 100 & 100 \\
\hline$a_{1}$ & $-88,7$ & $-182,8$ & $-251,4$ & $-242,6$ & $-357,3$ & $-572,9$ & $-469,3$ & $-396,1$ & $-577,6$ & $-684,3$ \\
\hline$a_{2}$ & 454,0 & 3781,6 & 4223,9 & 2148,9 & 5524,5 & 13660,0 & 7252,0 & $-1198,9$ & 8864,3 & 9248,4 \\
\hline$a_{3}$ & 4719,5 & $-47754,5$ & $-55634,7$ & $-4162,3$ & $-66543,0$ & $-219163,6$ & $-86741,4$ & 119100,6 & $-104209,1$ & $-86821,5$ \\
\hline$a_{4}$ & $-113190,0$ & 358207,8 & 481249,7 & $-129020,2$ & 526397,3 & 2134038,5 & 682209,7 & $-1781952,0$ & 805895,7 & 532016,2 \\
\hline$a_{5}$ & 957015,7 & $-1687800,5$ & $-2768878,8$ & 1470870,0 & $-2764903,7$ & $-13022337,6$ & $-3559002,7$ & 13594552,2 & $-4135421,6$ & $-2260576,2$ \\
\hline$a_{6}$ & $-4473462,2$ & 5132416,9 & 10680702,7 & $-7709733,4$ & 9728193,1 & 51049511,1 & 12413443,5 & $-61391738,5$ & 14195574,1 & 7125696,7 \\
\hline$a_{7}$ & 12559747,8 & $-10044517,7$ & $-27258807,6$ & 23187361,5 & $-22664547,0$ & $-128519111,3$ & $-28605887,8$ & 170587764,1 & $-32220275,1$ & $-16790157,4$ \\
\hline$a_{8}$ & $-21096551,5$ & 12180376,5 & 44059429,4 & $-40996252,7$ & 33535228,2 & 200843071,0 & 41770962,5 & $-286783820,3$ & 46384859,5 & 27432124,8 \\
\hline$a_{9}$ & 19564855,4 & $-8286347,9$ & $-40740040,3$ & 39687366,2 & $-28523653,2$ & $-177308022,0$ & $-34985276,1$ & 267836101,2 & $-38341325,4$ & $-26780575,1$ \\
\hline$a_{10}$ & $-7712384,0$ & 2399990,3 & 16372793,2 & $-16255502,5$ & 10614375,1 & 67563228,8 & 12792706,6 & $-106721471,3$ & 13851225,0 & 11487040,7 \\
\hline
\end{tabular}




\section{Appendix}

\section{EEP formula based on the J-model}

For an American put, the EEP is: $\varepsilon=\int_{0}^{T-t} e^{-r \omega} \int_{0}^{S_{(t-\omega)}^{*}}\left(r K-Q s_{\omega}\right) h_{S}\left(s_{\omega}\right) d s_{\omega} d \omega$, where $h_{S}\left(s_{\omega}\right)$ is the probability density function of the underlying asset price $S_{\omega}$ at a time $\mathrm{t}$ ranging from 0 to the option time to maturity. The underlying asset, distributing dividends at a rate $\mathrm{Q}$, follows a $J$-process defined by the following SDE: $\frac{d S_{t}}{S_{t}}=(r-Q) d t+\sigma \sqrt{d t} U_{t}$, where $U_{t}=\frac{W_{t}-E_{W}}{\sigma_{W}}$ with W following the J-Law (see Jerbi (2015), with expected value $E_{W}=E\left(W_{t}\right)$ and standard deviation $\sigma_{W}=\sqrt{V\left(W_{t}\right)}$.

Applying the Ito's lemma and replacing $U_{t}$ by its expression, and integrating from 0 to $\omega$, we have: $\operatorname{Ln}\left(\frac{S_{\omega}}{S_{t}}\right)=\left(r-Q-\frac{\sigma^{2}}{2}\right) \omega-\frac{\sigma}{\sigma_{W}} E_{W} \sqrt{\omega}+W_{\omega} \frac{\sigma}{\sigma_{W}} \sqrt{\omega}$. If we put:

$$
\left\{\begin{array} { l } 
{ a = ( r - Q - \frac { \sigma ^ { 2 } } { 2 } ) \omega - \frac { \sigma } { \sigma _ { W } } E _ { W } \sqrt { \omega } } \\
{ b = \frac { \sigma } { \sigma _ { W } } \sqrt { \omega } }
\end{array} \text { we can write } \left\{\begin{array}{l}
S_{\omega}=S_{t} e^{a+b W_{\omega}} \\
W_{\omega}=\frac{1}{b}\left\{\operatorname{Ln}\left(\frac{S_{\omega}}{S_{t}}\right)-a\right\}
\end{array}\right.\right.
$$

As $S_{\omega}$ is a strictly monotonous function of $W_{\omega}$, we can write $h\left(s_{\omega}\right) d s_{\omega}=g\left(w_{\omega}\right) d w_{\omega}$. If we put $H(\omega)=\int_{0}^{S_{(\tau-\omega)}^{*}}\left(r K-Q s_{\omega}\right) h_{S}\left(s_{\omega}\right) d s_{\omega}$, the premium is then $\varepsilon=\int_{0}^{T-t} e^{-r \omega} H(\omega) d \omega$.

In replacing $s_{\omega}$ by its expression as a function of $w_{\omega}$, we get: $H(\omega)=$ $\int_{-\infty}^{w_{(\tau-\omega)}^{*}}\left(r K-Q S_{t} e^{a+b w_{\omega}}\right) g\left(w_{\omega}\right) d w_{\omega}$.

$H(\omega)$ can be written as the difference : $H(\omega)=H_{1}(\omega)-H_{2}(\omega)$ with :

$$
\left\{\begin{array}{l}
H_{1}(\omega)=r K \int_{-\infty}^{w_{(\tau-\omega)}^{*}} f\left(w_{\omega}\right) d w_{\omega} \\
H_{2}(\omega)=Q S_{t} \int_{-\infty}^{w_{(\tau-\omega)}^{*}} e^{a+b w_{\omega}} \frac{e^{-\frac{1}{2} w^{2}}}{\operatorname{Jer}(\lambda, \theta) \sqrt{2 \pi}} N\left(\lambda w_{\omega}+\theta\right) d w_{\omega}
\end{array}\right.
$$

If we name $\mathrm{F}$ the cumulative function of the $J$-law, we have:

$$
\left\{\begin{array}{l}
H_{1}(\omega)=r K F\left(w_{(\tau-\omega)}^{*}, \lambda, \theta\right) \\
H_{2}(\omega)=Q S_{t} e^{a+\frac{b^{2}}{2} \int_{-\infty}^{w_{(\tau-\omega)}^{*}} \frac{e^{-\frac{1}{2}\left(w_{\omega}-b\right)^{2}}}{\operatorname{Jer}(\lambda, \theta) \sqrt{2 \pi}} N\left(\lambda w_{\omega}+\theta\right) d w_{\omega}}
\end{array}\right.
$$

We put $Z_{\omega}=W_{\omega}-b, Z_{\omega}$ follows the $J$-law such as: $Z_{\omega} \rightarrow J(\lambda, \lambda b+\theta)$. Hence, we have:

$$
\left\{\begin{array}{l}
H_{1}(\omega)=r K F\left(w_{(\tau-\omega)}^{*}, \lambda, \theta\right) \\
H_{2}(\omega)=Q S_{t} e^{a+\frac{b^{2}}{2} F\left(z_{(\tau-\omega)}^{*}, \lambda, \lambda b+\theta\right)} \text { with } z_{(\tau-\omega)}^{*}=w_{(\tau-\omega)}^{*}-b
\end{array}\right.
$$

If we call $\Phi=-\frac{\sigma^{2} \omega}{2}\left(1-\frac{1}{\sigma_{W}^{2}}\right)-\frac{\sigma \sqrt{\omega}}{\sigma_{W}} E_{W}$, we have $a+\frac{b^{2}}{2}=r \omega-Q \omega+\Phi$. Hence, $H(\omega)$ can be written as follows: $H(\omega)=r K F\left(w_{(\tau-\omega)}^{*}, \lambda, \theta\right)-Q S_{t} e^{r \omega-Q \omega+\Phi} F\left(z_{(\tau-\omega)}^{*}, \lambda, \lambda b+\theta\right)$.

We deduce the expression of the EEP: $\varepsilon=\int_{0^{T-t}} G(\omega) d \omega$ with $G(\omega)=\left\{r K e^{-r \omega} F\left(w_{(\tau-\omega)}^{*}, \lambda, \theta\right)-Q S_{t} e^{-Q \omega+\Phi} F\left(z_{(\tau-\omega)}^{*}, \lambda, \lambda b+\theta\right)\right\}$ 
where $w_{(\tau-\omega)}^{*}=\frac{\operatorname{Ln}\left(\frac{S_{W}}{S_{t}}\right)-\left(r-Q-\frac{\sigma^{2}}{2}\right) \omega+\frac{\sigma}{\sigma_{W}} E_{W} \sqrt{\omega}}{\frac{\sigma}{\sigma_{W}} \sqrt{\omega}}$ and $z_{(\tau-\omega)}^{*}=w_{(\tau-\omega)}^{*}-b$.

We notice that:

$$
\left\{\begin{array}{l}
z_{(\tau-\omega)}^{*}=-\sigma_{W} d_{1, \omega}+E_{W} \\
w_{(\tau-\omega)}^{*}=-\sigma_{W} d_{2, \omega}+E_{W}
\end{array} \text { with } d_{1, \omega}=\frac{\operatorname{Ln}\left(\frac{S_{t}}{S_{W}}\right)+\left(r-Q+\frac{\sigma^{2}}{2}\right) \omega}{\sigma \sqrt{\omega}} \text { et } d_{2, \omega}=d_{1, \omega}-\sigma \sqrt{\omega}\right.
$$

If we set $\lambda=0$, we get the EEP formula for an American put related to BS-am model:

$$
\varepsilon=\int_{0}^{\tau}\left[r K e^{-r \omega} N\left(-d_{2, \omega}\right)-Q S_{t} e^{-Q \omega} N\left(-d_{1, \omega}\right)\right] d \omega
$$

\section{Acknowledgement}

I have achieved all the work by myself and I have no acknowledgement to mention in the Acknowledgement section.

\section{Competing interest}

The author declares that he has no competing interests.

Received: 22 February 2016 Accepted: 28 November 2016

Published online: 07 December 2016

\section{References}

Black F, Scholes M (1973) The pricing of options and corporate Liabilities. J Polit Econ 81(May-June):637-654

Brennan M, Schwartz E (1977) The valuation of American put options. J Financ 32:449-462

Broadie M, Glasserman P (1997) Pricing American-style securities using simulation. J Econ Dyn Control 21:1323-1352

Carr P, Wu L (2004) Time-changed levy processes and option pricing. J Financ Econ 17(1):113-141

Clarke N, Parrott K (1999) Multigrid for American option pricing with stochastic volatility. Applied Mathematical Finance 6:177-195

Detemple J, Rindisbacher M (2005) Closed-form solutions for optimal portfolio selection with stochastic interest rates. Math Financ 15:539-568

Detemple J, Tian W (2002) The valuation of American options for a class of diffusion processes. Manag Sci 48:917-937 Garman MB (1976) A general theory of asset valuation under diffusion state processes, research program in finance, Working papers 50, University of California at Berkeley

Haugh M, Kogan L (2004) Pricing American options: a duality approach. Operation Research 52:258-270

Heston SL (1993) Closed form solution for options with stochastic volatility with application to bonds and currency options. Rev Financ Stud 6(2):327-343

Huang J, Subrahmanyam M, Yu G (1996) Pricing and hedging American options: a recursive integration method. Rev Financ Stud 9:277-300

Hull J, White A (1987) The pricing of options on assets with stochastic volatilities. J Financ 42(2):281-300

Ikonen S, Toivanen J (2007) Efficient numerical methods for pricing American options under stochastic volatility. Numerical Methods for Partial Differential Equations 24:104-126

Jerbi Y (2011) New statistical law-named J-law and its features. Far East Journal of Applied Mathematics 50(1):41-56

Jerbi Y (2015) A New closed form solution as an extension of the Black \& Scholes formula allowing smile curve plotting. Quantitative Finance 15(12):2041-2052

Jerbi Y, Kharrat M (2014) Conditional expectation determination based on the J-process using Malliavin calculus applied to pricing American options. J Stat Comput Simul 84(11):1-9

Ju N (1998) Pricing an American Option by approximating its early exercise boundary as a multi piece exponential function. Rev Financ Stud 11(3):627-646

Kim IJ (1990) The analytical valuation of American options. Rev Financ Stud 3:547-572

Kwok Y-K (1998) Mathematical model of financial derivatives, Springer

Longstaff F, Schwartz E (2001) Valuing American options by simulation: a simple least-squares approach. Rev Financ Stud 14:113-147

Rogers L (2002) Monte Carlo valuation of American options. Math Financ 12:271-286

Stein EM, Stein JC (1991) Stock price distributions with stochastic volatility: an analytic approach. Rev Financ Stud 4(4): $727-752$

Sullivan M (2000) Valuing American put options using Gaussian quadrature. Rev Financ Stud 13:75-94

Wiggins JB (1987) Option values under stochastic volatility: theory and empirical estimates. J Financ Econ 19(2):351-372

Zauderer E (1989) Partial differential equations of applied mathematics, 2nd edn. John Wiley and Sons, New York 